\title{
Genome-wide analysis, identification, evolution and genomic organization of Dehydration Responsive Element Binding (DREB) gene family in Solanum tuberosum
}

\author{
Nida Mushtaq ${ }^{1}$, Faiza Munir ${ }^{\text {Corresp., } 1}{ }^{1}$, Alvina Gul ${ }^{1}$, Rabia Amir ${ }^{1}$, Rehan Zafar Paracha ${ }^{2}$ \\ 1 Department of Plant Biotechnology, Atta-ur-Rahman School of Applied Biosciences, National University of Sciences and Technology, Islamabad, Pakistan \\ 2 Research Centre for Modelling \& Simulation, National University of Sciences and Technology, Islamabad, Pakistan \\ Corresponding Author: Faiza Munir \\ Email address: faiza.munir@asab.nust.edu.pk
}

Background: Dehydration Responsive Element Binding (DREB) gene family plays a crucial role as transcription regulators and enhances plant tolerance to abiotic stresses. Although the DREB gene family has been identified and characterized in many plants, knowledge about it in Solanum tuberosum (Potato) is limited. Results: In the present study, StDREB gene family was comprehensively analyzed using bioinformatics approaches. We identified 66 StDREB genes through genome wide screening of Potato genome based on the AP2 domain architecture and amino acid conservation analysis (Valine at position $14^{\text {th }}$ ). Phylogenetic analysis divided them into six distinct subgroups (A1-A6). The categorization of StDREB genes into six subgroups was further supported by gene structure and conserved motif analysis. Potato DREB genes were found to be distributed unevenly across 12 chromosomes. Gene duplication proved that StDREB genes experienced tandem and segmental duplication events which led to the expansion of the gene family. The Ka/Ks ratios of the orthologous pairs also demonstrated the StDREB genes were under strong purification selection in the course of evolution. Interspecies synteny analysis revealed 45 and 36 StDREB genes were orthologous to Arabidopsis and Solanum lycopersicum, respectively. Moreover, subcellular localization indicated that StDREB genes were predominantly located within the nucleus and the StDREB family's major function was DNA binding according to gene ontology (GO) annotation. Conclusions: This study provides a comprehensive and systematic understanding of precise molecular mechanism and functional characterization of StDREB genes in abiotic stress responses and will lead to improvement in Solanum tuberosum. 
1 Genome-wide analysis, identification, evolution and genomic organization of Dehydration

2 Responsive Element Binding (DREB) gene family in Solanum tuberosum

3

4

5

6

7

\author{
Nida Mushtaq ${ }^{1}$, Faiza Munir1 ${ }^{*}$, Alvina Gul${ }^{1}$, Rabia Amir ${ }^{1}$, Rehan Zafar Paracha² \\ ${ }^{1}$ Department of Plant Biotechnology, Atta-ur-Rahman School of Applied Biosciences, \\ National University of Sciences and Technology, Islamabad, Pakistan \\ ${ }^{2}$ Research Centre for Modelling \& Simulation, National University of Sciences and \\ Technology Islamabad, Pakistan.

\section{*Corresponding author's} \\ Faiza Munir \\ E-mail: faiza.munir@asab.nust.edu.pk \\ Telephone: +92-51-90856146
}

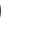

(1)

22




\section{Abstract}

Background: Dehydration Responsive Element Binding (DREB) gene family plays a crucial role as transcription regulators and enhances plant tolerance to abiotic stresses. Although the DREB gene family has been identified and characterized in many plants, knowledge about it in Solanum tuberosum (Potato) is limited.

Results: In the present study, StDREB gene family was comprehensively analyzed using bioinformatics approaches. We identified 66 StDREB genes through genome wide screening of Potato genome based on the AP2 domain architecture and amino acid conservation analysis (Valine at position $14^{\text {th }}$ ). Phylogenetic analysis divided them into six distinct subgroups (A1-A6). The categorization of StDREB genes into six subgroups was further supported by gene structure and conserved motif analysis. Potato DREB genes were found to be distributed unevenly across 12 chromosomes. Gene duplication proved that StDREB genes experienced tandem and segmental duplication events which led to the expansion of the gene family. The $\mathrm{Ka} / \mathrm{Ks}$ ratios of the orthologous pairs also demonstrated the StDREB genes were under strong purification selection in the course of evolution. Interspecies synteny analysis revealed 45 and 36 StDREB genes were orthologous to Arabidopsis and Solanum lycopersicum, respectively. Moreover, subcellular localization indicated that StDREB genes were predominantly located within the nucleus and the StDREB family's major function was DNA binding according to gene ontology (GO) annotation.

Conclusions: This study provides a comprehensive and systematic understanding of precise molecular mechanism and functional characterization of StDREB genes in abiotic stress responses and will lead to improvement in Solanum tuberosum.

Keywords: DREB, Solanum tuberosum, Bioinformatics, Phylogeny, Gene Duplication, GO annotation

Abbreviations: DRE, dehydration responsive element; CRT, C- repeat; AP2, APETALA2 
65

66

67

68

69

70

71

72

73

74

75

76

77

78

79

80

81

82

83

84

85

86

87

88

89

90

91

92

93

94

95

96

97

98

99

100

101

102

103

104

105

106

107

108

109

\section{INTRODUCTION}

Dehydration responsive element binding (DREB) is considered as one of the largest and best studied gene family involved in the abiotic stress mitigation by regulating the expression of genes involved in ABA-independent stress tolerance pathway (Lata \& Prasad 2011). DREB belongs to the AP2 multigene family and possess a single conserved AP2 domain (Dietz et al. 2010). The DREB gene family is characterized by the presence of valine (V) at position 14th and glutamic acid (E) at position 19th respectively within the conserved AP2 domain (Zhou et al. 2010). The AP2 domain consists of 60-70 conserved amino acid residues, indispensable for plant's stress and defense response mechanisms (Wu et al. 2015). The AP2 domain comprises seven key amino acids that are necessary for DRE binding: one $\mathrm{V}$ residue, four $\mathrm{R}$ residues, and two $\mathrm{W}$ residues (Allen et al. 1998). In addition, a conserved Serine/Threonine rich region is located adjacent to the AP2 domain, which is responsible for phosphorylation of the DREB genes (Shen et al. 2003). Based on their structural characteristics, DREB genes can be further divided into six subgroups: A1-A6 (Zhou et al. 2010). DREB transcription factors are one of the most promising regulons for abiotic stress tolerance in plants that directly interact with DRE/CRT, which consist of core motifs ACCGAC/GCCGAC (Sharoni et al. 2011; VazquezHernandez et al. 2017). These sequences are found in the promoter regions of drought and cold responsive genes for example KIN1 and KIN2 (cold responsive) (Kimotho et al. 2019), RD29A (drought responsive) (Zhao et al. 2013), LEA (Late Embryogenesis Abundant) (Liang et al. 2019), COR15A and COR15B (cold responsive) (Yu et al. 2018).

DREB gene family members are widely involved in abiotic stress responses such as drought, salt, cold, and heat (Du et al. 2018; Kudo et al. 2017; Wu et al. 2018). DREB genes were first identified in Arabidopsis. AtDREB1 and AtDREB2 can function as two independent proteins in two distinct signal transduction pathways under cold and dehydration stress, respectively (Liu et al. 1998). In Arabidopsis, studies have shown that ABA signaling does not normally mediate the expression of the A1 and A2 subgroups. On the contrary, ABI4, the only member of A3 subgroup is involved in sugar and ABA signaling (Shkolnik-Inbar \& Bar-Zvi 2011). A4 subgroup includes the most studied members namely TINY which functions in drought tolerance and HARDY which participates in both salinity and drought tolerance (Xie et al. 2019). Drought and cold stress induce RAP2.1, member of A5 subgroup, in Arabidopsis (Dong \& Liu 2010). RAP2.4 and RAP2.4B genes in the A6 subgroup are responsive to drought, heat and salinity stress respectively (Rae et al. 2011). DREB genes are reported to impart enhanced drought tolerance in many plant species. Overexpression of DREB1A led to heightened drought tolerance in Arabidopsis and tobacco. A similar phenotype is reported in wheat using the RD29A promoter. Likewise, DREB gene from cotton conferred enhanced tolerance to drought, heat and cold stress when expressed in wheat (Rabara et al. 2014). Constitutive overexpression of CsDREB gene led to enhanced drought and salt tolerance in transgenic Arabidopsis (Wang et al. 2017).

Subsequently, a number of DREB genes have been identified and characterized in a wide variety of plant species such as Arabidopsis (Hwang et al. 2012), rice (Dubouzet et al. 2003), bell pepper (Hong \& Kim 2005), soybean (Chen et al. 2007), pearl millet (Agarwal et al. 2007), wheat (Egawa et al. 2006; Lucas et al. 2011 ), maize (Feng et al. 2003; Liu et al. 2013), chrysanthemum (Yang et al. 2009), tomato (Guo \& Wang 2011; Hichri et al. 2016), lettuce (Park et al. 2020). This depicts their involvement in different abiotic stress responses. For instance, transgenic expression of DREB3a from a forage grass Leymus chinensis led to improved drought and salt

Peer) reviewing PDF | (2021:04:60076:1:0:NEW 20 May 2021) 
110 tolerance in Arabidopsis (Xianjun et al. 2011). Another study reported enhanced drought and 111 freezing stress tolerance by constitutive overexpression of Arabidopsis DREB1B gene in 112 transgenic potato (Movahedi et al. 2012). Functional analysis of Broussonetia papyrifera DREB2 gene suggested its participation in drought and salinity stress responses. Furthermore, transgenic expression of BpDREB2 gene in Arabidopsis demonstrated enhanced salt and cold stress (Sun et al. 2014). Studies on Medicago truncatula revealed the role of DREB gene members both in freezing and cold stress (Shu et al. 2016). A novel DREB gene has been reported in maize namely ZmDBF3 which exhibits positive regulation relationship under salinity, drought, heat and cold stress (Zhou et al. 2016).

The distribution of DREB genes varies greatly between plants. Polyploidization is thought to have a major contribution in genome evolution and plant diversity (Wendel et al. 2016). Gene diversification is greatly aided by gene duplication events, which results in the generation of novel genes necessary for plant evolution (McKain et al. 2016). According to a recent study, polyploidization has played vital roles in the expansion of the DREB gene family specifically for plants that have undergone recent whole genome duplication (WGD) events such as t for Commelinid- specific and $r$ for Poaceae (Wang et al. 2019). The DREB gene family has expanded significantly, allowing for more extensive and complex functional distinction. For instance, 30 DREB genes have been characterized in mung bean, five of which are highly expressed under drought stress (Labbo et al. 2018). In barley, 41 DREB genes have been discovered, many of which are expressed in drought and salt stress (Guo et al. 2016). Studies on Musa acuminata and Musa balbisiana revealed 81 and 99 DREB genes, respectively (Lakhwani et al. 2016). A large number of DREB genes (210) haven been identified in wheat genome. Over-expression of TaDREB3-AI displayed enhanced tolerance to heat, dehydration, and salinity stresses (Niu et al. 2020).

Potato, which originated from the Andean regions of Bolivia and Peru (Davies Jr et al. 2005), is the third most significant agricultural crop worldwide after wheat and rice. Potato is highly adaptable to a wide range of ecosystems. According to FAO, over 388 million tons of potatoes were produced annually with consumption of over 239 million tons in 2017 (Handayani et al. 2019). Being a wholesome food, potato is rich in vitamins, minerals and complex carbohydrates (Hussain 2016 ). However, potato is highly sensitive to various types of stress thus affecting its sustainable production. The key factors that severely affect potato's yield are abiotic stresses, including drought, low temperature, heat and salinity (Dahal et al. 2019). In previous studies, characterization of two DREB genes (StDREB1 and StDREB2) showed a remarkable increase in their expression by salinity stress. The ectopic expression of these genes in potato conferred enhanced tolerance to salinity stress in transgenic potato lines (Bouaziz et al. 2015b; Bouaziz et al. 2012). Furthermore, overexpression of StDREB1 and StDREB2 led to enhanced drought tolerance in transgenic potato lines (Bouaziz et al. 2015a). In addition, overexpression of DREB transcription factors imparted increased cadmium $(\mathrm{Cd})$ stress tolerance in transgenic potato cultivars (Charfeddine et al. 2017). Despite overexpression, little is known about characterization of the DREB gene family in S. tuberosum. Given the vital role that DREB genes play in plant's abiotic stress tolerance, it is essential to identify and study the DREB gene family in the potato genome. 
153 In the present study, we identified all potential DREB genes encoded in the Solanum tuberosum 154 genome. Further, we performed bioinformatics analysis for classification of DREB genes into 155 different subgroups, presence of characteristic motifs, exon/intron organization, chromosomal 156 distribution, and gene duplication events. Finally, we analyzed homology of DREB genes with 157 A. thaliana and S. lycopersicum, functional diversity, and subcellular localization of StDREB 158 genes. The results gained herein will provide useful insights for future studies on functional 159 characterization of DREBs in Potato.

160

161

162

163

164

165

166

167

168

169

170

171

172

173

174

175

176

177

178

179

180

181

182

183

184

185

186

187

188

189

190

191

192

193

194

\section{MATERIALS AND METHODS}

\section{Retrieval and Identification of DREB Genes in Solanum tuberosum Genome}

To perform a comprehensive identification of the DREB gene family members in $S$. tuberosum, the amino acid sequences of Arabidopsis DREB proteins were retrieved from TAIR database (https://www.arabidopsis.org/) (Huala et al. 2001) and used as queries for BLASTp homology search against the Potato genome v4.03 with an e-value of $1 \times 10^{-5}$ in Sol Genomics Network (SGN) (https://solgenomics.net/) (Mueller et al. 2005) and Phytozome v12.1 database (https://phytozome.jgi.doe.gov/pz/portal.html) (Goodstein et al. 2012), respectively. Since DREB gene family consists of only one conserved AP2 domain, all retrieved amino acid sequences of potato were scanned for the presence of AP2 domain using PFAM (http://pfam.xfam.org/) (Mistry et al. 2021) and SMART (http://smart.embl-heidelberg.de/) (Letunic \& Bork 2018) programs. Furthermore, amino acid conservation analysis was performed by alignment of the AP2 domain for each filtered DREB protein using MEGA X. The candidate protein sequences were validated for the presence of valine $(\mathrm{V})$ at position $14^{\text {th }}$ and glutamic acid (E) at position $19^{\text {th }}$, especially valine, which has been a characteristic feature for DREB gene selection. Amino acid positions were also assessed by ScanProsite server (https://prosite.expasy.org/scanprosite/) (Hulo et al. 2006). Additionally, physiochemical properties of DREB proteins such as protein length, molecular weight $(\mathrm{kDa})$ and isoelectric point $(\mathrm{pI})$ were computed by ExPASy ProtParam tool (https://web.expasy.org/protparam/) (Gasteiger et al. 2003).

\section{Phylogenetic Analysis StDREB proteins}

Full length amino acid sequences of AtDREB and StDREB proteins were aligned in order to construct the phylogenetic trees. Multiple sequence alignment (MSA) was executed by MUSCLE algorithm with default parameters. Following alignment, phylogenetic trees were constructed by neighbor-joining (NJ) method based on pairwise deletion and Poisson substitution model with 1000 bootstrap replicates using MEGA X software (http://www.megasoftware.net) (Kumar et al. 2018). Based on the classification schemes for AtDREB proteins, recently identified StDREB proteins were characterized into distinct subgroups.

\section{Gene Structure and Protein Motif Features}

Genomic and complete coding sequences (CDS) of each StDREB gene were downloaded from Phytozome v12.1 to analyze exon-intron structures. Gene Structure Display Server (GSDS 2.0) (http://gsds.gao-lab.org/) was utilized for a detailed graphical illustration of the exon-intron organization by comparing CDS sequences of the StDREB genes with their respective genomic sequences (Guo et al. 2007). The MEME version 5.3.2 (https://memesuite.org/meme/tools/meme) was employed to detect conserved motifs in $S$. tuberosum DREB proteins with the following parameters: (i) an optimal motif width of 6-50 
195

196

197

198

199

200

201

202

203

204

205

206

207

208

209

210

211

212

213

214

215

216

217

218

219

220

221

222

223

224

225

226

227

228

229

230

231

232

233

234

235

236

amino acids, (ii) zero or one occurrence per sequence, and (iii) a maximum number of motifs set to 15 (Bailey et al. 2015).

\section{In Silico Chromosomal Mapping, Gene Duplication Events and Synteny Analysis} Chromosomal position information of each StDREB gene was determined from Phytozome v12.1 database. Physical locations and relative distances of the StDREB genes were mapped on to their respective potato chromosomes using MapChart 2.2 Software (https://www.wur.nl/en/show/Mapchart.htm) (Voorrips 2002). To analyze gene duplication events two mechanisms of gene expansion were considered: tandem duplications and segmental duplications. Two gene pairs situated on same chromosomal fragment and segregated by five or fewer gene loci were regarded as tandem duplications. To assess the effect of selective pressure and divergence time of StDREB genes, the $\mathrm{Ka}$ (non-synonymous) and $\mathrm{Ks}$ (synonyms) values were computed using ToolKit Biologists Tools (TB tools) software (https://github.com/CJChen/TBtools) (Chen et al. 2020). The approximate divergence time was estimated using the formula $\mathrm{T}=\mathrm{Ks} / 2 \mathrm{x} * \mathrm{MYA}$, where $\mathrm{x}=6.56 \times 10^{-9}$ and $\mathrm{MYA}=10^{-6}$ (He et al. 2016). For synteny analysis, both the genomic and gff3 annotation files of $S$. tuberosum, A. thaliana and $S$. lycopersicum were extracted. Circos Plot and synteny images were constructed and visualized through advanced Circos and dual synteny plotter software (https://github.com/CJChen/TBtools) to examine segmental duplication gene pairs and orthologous gene conservation of StDREBs with other plants species respectively.

\section{Gene Ontology (GO) Annotation and Subcellular Localization Prediction}

Gene ontology annotation analysis of StDREB genes was conducted through Blast2Go software (https://www.blast2go.com/) (Conesa \& Götz 2008). Transcript sequence of each StDREB gene was uploaded to this software to determine biological processes (BP), cellular compartments (CC) and, molecular functions (MF). To execute Blast2Go functional annotation BLASTx search, InterPro Scan, mapping and annotation were carried out with default settings. The WoLF PSORT tool (https://wolfpsort.hgc.jp/) (Horton et al. 2007) and CELLO Online server v.2.5 (http://cello.life.nctu.edu.tw/) were used to predict the subcellular localization of DREB genes.

\section{RESULTS}

\section{Identification and Characterization of Putative DREB Genes in S. tuberosum Genome}

To identify all potential DREB gene family members in Potato, DREB protein sequences from the model plant Arabidopsis thaliana were used as queries in BLASTp homology search against S. tuberosum genome v4.03. A total of 66 DREB genes designated as StDREB1 to StDREB66 were identified in Potato by removing other redundant hits with insignificant e-value and identity percentage. Based on Phytozome v12.1 annotation, all of the StDREB genes belonged to dehydration responsive element binding protein and ethylene-response factor (Data S1). Detailed information of all the StDREB genes aligned with their respective Arabidopsis ortholog were predicted (Data S2). Later, StDREB genes were verified by using SMART and Pfam analysis in order to confirm the presence of one conserved AP2 domain as shown in Table 1 followed by amino acid conservation analysis. Valine has been indicated as the most significant amino acid for binding affinity whereas glutamic acid might have some flexibility among proteins. Among 66 StDREB proteins, 26 sequences presented only valine residue at the position 
$23714^{\text {th }}$, while 40 sequences exhibited both $\mathrm{V}$ and $\mathrm{E}$ at position $14^{\text {th }}$ and $19^{\text {th }}$ within the conserved 238 AP2 domain, respectively. StDREB1 to StDREB66 genes were subjected to further analysis in

239

240

241

242

243

244

245

246

247

248

249

250

251

252

253

254

255

256

257

258

259

260

261

262

263

264

265

266

267

268

269

270

271

272

273

274

275

276

277

278

279

order to assess their physiochemical characteristics. Analysis depicted that the amino acid lengths of 66 StDREB proteins varied greatly from 72 a.a residues (StDREB51) to 457 a.a residues (StDREB31) with different range of molecular weights from $8.3 \mathrm{kDa}$ to $52.29 \mathrm{kDa}$ and isoelectric points (pI) ranged from 4.17 (StDREB59) to 9.97 (StDREB51), respectively as provided in Data $\mathbf{S 2}$.

\section{Phylogeny and Group Classification of StDREB Genes}

To investigate the evolutionary relationship and sequence homology between Arabidopsis and $S$. tuberosum DREB protein sequences, a Neighbor- Joining (NJ) phylogenetic method with 1000 bootstrap replicates was used to generate a phylogenetic tree for DREB proteins among $S$. tuberosum (66 DREB proteins) and A. thaliana (56 DREB proteins) (Data S3). The phylogenetic tree was constructed followed by multiple sequence alignment (MSA) of AP2 domain coding protein sequences present in potato DREB sequences. All 66 genes of DREB family in potato were categorized into six subgroups referred to as A1, A2, A3, A4, A5 and A6 with reference to the classification of AtDREBs as shown in Fig. 1.

The largest subgroup A6 consisted of 19 members while the smallest subgroup A3 consisted of only 2 StDREB members. A total of $11,8,18$ and 8 protein members were assigned to A1, A2, A4 and A5 subgroups, respectively.

The conserved amino acid sequence present in AP2 domain of StDREB members had valine at position $14^{\text {th }}$ and glutamic acid at position $19^{\text {th }}$ but some members only had valine which plays an important role in identification of the DREB gene family's various DNA binding sites. The phylogenetic tree revealed that StDREB members (StDREB50, StDREB52, StDREB39, StDREB40, StDREB56, StDREB65, StDREB61, StDREB66, and StDREB62) belonging to group 6 exhibited greater homology with each other rather than with Arabidopsis DREB sequences due to high structure similarity among them which was further investigated by gene structural analysis and conserved motifs identification in potato.

\section{Gene Structure and Motif Composition of StDREB Members}

The organization of introns and exons is pivotal in the evolution of gene families. Gene structure analysis was conducted through aligning the cDNA and gDNA sequences (Data S4) to gain further intuition into the structural similarity and divergence of DREB genes in Potato. We also constructed a phylogenetic tree as shown in Fig. 2 but using only full-length protein sequences of StDREB genes to relate it with exon/intron distribution and motif composition. Results showed that eight StDREB genes $(21,34,60,20,13,39,40$, and 52) had one intron with the exception of StDREB56 which contained 3 introns in its coding region while other fifty-seven StDREB genes had only exons in their coding region as shown in Fig. 2B. MEME motif detection web software was utilized to recognize the conserved motifs to further understand the diversification in StDREB members of Potato Fig. 2C. We found fifteen conserved motifs with different amino acid range from 9-50. Motif 12 was the shortest with 9 amino acid residues (DDDMSLWSY) while motif 15 was the longest with 50 amino acid residues (NNYJPYGFYPAVQYAEDISQNPQHSIQKQTFDDNYGFLDGETTKASGMIW). Motif composition in different subgroups was different (Figs. S1-S2). Motifs 1 and 2 were found within the AP2 domain. Majority of the StDREB proteins observed motif 1 and motif 2 with

Peer) reviewing PDF | (2021:04:60076:1:0:NEW 20 May 2021) 
280

very few exceptions. Motif 13 was unique to three $\operatorname{StDREB}(5,23$ and, 57) members belonging to A1 subgroup. Motif 15 was exclusively present in A3 subgroup $(63,64)$, whereas motif 8 was found both in A3 subgroup and seven out of eight (7, 9, 10, 13, 15, 29, and 31) members of A2 subgroup proposing that proteins in these subgroups may possibly share a specific function. Motif 9 was only encountered in five StDREB protein sequences $(11,22,24,28$, and 46) of the A4 subgroup. Motif 10, motif 11, and motif 14 were detected only within some members of A6 subgroup. Majority of StDREB proteins within the same subgroup exhibited similar intron-exon structure and motif compositions which supported the phylogenetic analysis of DREB gene family, while the difference among the distinct subgroups directed their diverse roles. The similarities and structural variations among these motifs can be studied further to provide new insights. Most StDREBs classified in the same subgroup generally had a similar motif composition and might have similar functions. Details of 15 conserved motifs are summarized in Data S5.

\section{Chromosomal Localization and Gene Duplication Analysis of StDREBs}

Chromosomal positions of all StDREB genes were obtained from Phytozome database v.12.1. Physical location of all the identified StDREB genes were mapped by using MapChart 2.2 software on to their corresponding chromosomes as depicted in Fig. 3. Chromosomal localization indicated that the 66 StDREB genes were heterogeneously distributed onto 12 chromosomes across the Potato genome which indicated that genetic variation occurred during the evolutionary process. Largest numbers of StDREB genes i.e. 13 were positioned on chromosome 3 (chr03). Conversely, chromosome 2 (chr02) and chromosome 9 (chr09) had the least number of StDREB genes i.e. 1. Two chromosomes (chr04 and chr11) hosted 5 StDREB genes. In addition, chromosome 8 (chr08) harbored 11 StDREB genes, chromosome 1 had 7 StDREB genes, chromosome 5 (chr05) and chromosome 7 (chr07) had 3 and 2 StDREB genes, respectively.

Tandem and segmental duplications contribute to the expansion of new gene family members and novel functions in the evolution of plant genome. To investigate gene duplication events within the potato genome, we investigated tandem and segmental duplications during evolution of StDREB gene family. A total of 10 StDREB gene pairs were confirmed to be tandem duplications. According to gene duplication analysis, it was observed that chr01, chr10, chr11 and chr12 each experienced one tandem duplicated gene pair while chr3 and chr8 harbored three tandem duplicated gene pairs illustrated as red lines in Fig. 3. Besides tandem duplication, 8 segmental duplication events were identified by constructing Circos Plot illustrated as red color in Fig. 4. To determine the selective evolutionary pressure on StDREB gene divergence after duplication, Ka and Ks values were computed for the duplicated StDREB gene pairs using KaKs calculator. Generally, KaKs $=1$ implies neutral selection, $\mathrm{Ka} / \mathrm{Ks}>1$ implies positive selection and, $\mathrm{Ka} / \mathrm{Ks}<1$ implies purification selection. Each duplicated $\mathrm{StDREB}$ gene pair had $\mathrm{Ka} / \mathrm{Ks}<1$, which was indicative of purification selection during evolution. Furthermore, duplication events of 18 gene pairs were estimated to have occurred between 6.15 and 223.0 million years ago (Data S6).

\section{Synteny Relationship of StDREB Genes}

To further infer the evolutionary relationship, DREB genes were compared to identify orthologous StDREB gene pairs between S. tuberosum, A. thaliana and S. lycopersicum. According to the synteny analysis, 36 of 66 StDREB genes had collinear gene pairs in A. 
324

325

326

327

328

329

330

331

332

333

334

335

336

337

338

339

340

341

342

343

344

345

346

347

348

349

350

351

352

353

354

355

356

357

358

359

360

361

362

363

364

365

thaliana and 45 of 66 StDREB genes had corresponding orthologs in S. lycopersicum, respectively as provided in Data S7. The syntenic maps showed high evolutionary homology relationship of StDREB genes with $A$. thaliana and $S$. lycopersicum DREB genes implying that they might have related functions as depicted in Fig. 5.

\section{Gene Ontology (GO) Annotation and Subcellular Localization Prediction of StDREB Genes}

To explore the functions of StDREB genes in different biological processes, molecular functions and cellular compartment building, GO functional annotation was performed as depicted in Fig. 6 and gene ontology number was also identified during the analysis as provided in Data S8. Biological processes showed that the StDREB genes were involved in cell metabolism, defense response and ethylene activated signaling. Majority of the StDREB genes were involved in sequence specific DNA-binding functions and transcriptional regulation, indicating their significance as a transcription factor for potato growth and development, as well as regulating the expression of abiotic stress responsive genes. The cellular compartment study revealed that most of the StDREB genes were concentrated in cell nucleus with the exception of StDREB47 and StDREB53 which were localized in cytoplasm. In addition, some StDREB genes were found in cell membrane to control the signal transduction inside the cell.

To predict the subcellular localization of StDREB proteins, two different tools were employed. Results confirmed that StDREB proteins were predominantly localized in the nucleus followed by mitochondria and chloroplast, respectively as indicated in the heat map (Fig. 7; Data S9.

\section{DISCUSSION}

Potato is one of the world's most important crops, providing food to over 100 countries. Potato is vulnerable to several abiotic stresses, and its steady development is jeopardized by recurrent stress outbreaks (Mirzaei et al. 2020). The DREB gene family plays an important role in plant's abiotic stress signaling. DREB gene family is well known for its highly conserved AP2 domain that can specifically bind to the DRE/CRT cis-acting elements to activate expression of several stress tolerance genes, thereby enhancing plant tolerance (Chen et al. 2016b). With the availability of whole-genome sequence, members of the DREB gene family have been identified in many plant species. Until now, bioinformatic analysis of DREB gene family has not been reported in potato. Therefore, our current study furnished a detailed data on genome-wide characterization of DREB genes in Solanum tuberosum. By using Arabidopsis thaliana DREB genes as query sequences, we identified 66 putative DREB genes in S. tuberosum (Data S2) and categorized them into six subgroups corresponding to $A$. thaliana DREB orthologs. The amino acid conservation (valine at 14th and glutamic acid at 19th) confirmed the presence of DREB genes in S. tuberosum genome.

The genome-wide analysis revealed the difference in number of genes and genome size of $S$. tuberosum and A. thaliana. The identified number of DREB genes in S. tuberosum (66) is greater than that in Arabidopsis (56) which may be due to the difference in their genome sizes. When compared to previous studies, greater numbers of DREB genes were reported in S. spontaneum (110) (Huang et al. 2020), P. trichocarpa (75) (Chen et al. 2013), soybean (73) (Zhou et al. 2020), and malus (68) (Zhao et al. 2012), but less DREB genes were found in sesame (41) (Dossa et al. 2016), maize (51) (Du et al. 2014), mulberry (30) (Liu et al. 2015), grape (38) 
366

367

368

369

370

371

372

373

374

375

376

377

378

379

380

381

382

383

384

385

386

387

388

389

390

391

392

393

394

395

396

397

398

399

400

401

402

403

404

405

406

407

408

409

410

(Zhao et al. 2014), common bean (54) (Konzen et al. 2019), Phyllostachys edulis (27), and pineapple (20) (Chai et al. 2020). In order to comprehend the potential functions of proteins, it is inevitable to compute the physiochemical characteristics of plant protein families (Salih et al. 2019). The DREB proteins in potato demonstrated diversification in terms of physiochemical properties, which indicated that these genes might play various roles in plant development and defense response. The lengths of all DREB proteins had a range of 72-457 amino acid residues. The molecular mass largely varied between 8.3 and $52.29 \mathrm{kDa}$, and the isoelectric point ranged from 4.17 to 9.97 (Data S2). Moreover, our phylogenetic analysis of $S$. tuberosum and $A$. thaliana DREB proteins demonstrated that the six subgroups (A1-A6) identified previously in Arabidopsis (Akhtar et al. 2012) were also present in $S$. tuberosum. The total number of genes for each group was $11,8,2,18,8$, and 19, corresponding to the A1 to A6 subgroups. This distribution was quite different from $A$. thaliana due to different genetic patterns in both plants (Fig. 1). Gene number similar to StDREB A3 subgroup had been reported in Salix arbutifolia (2) (Rao et al. 2015) and P. trichocarpa (2). However, 18 DREB genes were categorized in A3 subgroup of foxtail millet (Shi et al. 2018), five in S. spontaneum, one in mulberry, one in Phaseolus vulgaris, and zero in pineapple.

To gain insights into the structural diversity of the StDREB genes, their gene structures were analyzed. Formerly, it was thought that DREB genes only contain coding region exons, without any intron as in Arabidopsis. But, later studies revealed that DREB genes contain both the exon and intron in their gene structure. This finding was further confirmed by gene structure analysis in wheat (Sazegari \& Niazi 2012), rice (Matsukura et al. 2010) and maize. Our study demonstrated nine StDREB genes consist of intronic regions along with exons. Out of nine StDREB genes, StDREB56 contain three introns whereas remaining 8 genes contain one intron only. While $76 \%$ of the StDREB genes showed a single exon (Fig. 2B). A previous study conducted on pineapple had also demonstrated highest number of introns as three in one of its 20 DREB genes. There was a strong connection of exon/intron structure between $S$. tuberosum and other species (S. spontaneum, soybean, pineapple, and populas) due to the presence of both exon and intron. Also, some studies showed that a compact gene structure with few or no introns enhanced timely response to various abiotic stresses in plants (Jeffares et al. 2008). The variation found in gene structure of StDREB genes elucidates the functional diversification which might be due to climatic or evolutionary changes in the plant genome profile (Li et al. 2020). Recent studies revealed the presence of some conserved motif sequences in transcription factors of $F$. tataricum located on the same chromosome (Liu et al. 2019), similar profile was observed in $A$. thaliana (Sakuma et al. 2002) which may be due to polyploid changes that occurred in the genome. Same results were obtained in S. tuberosum DREB genes through identification of conserved motif sequences. We identified 15 conserved motifs in StDREB genes along with the conserved AP2 domain (Fig. 2C). The AP2 domain has one alpha helix and three beta sheets at the N-terminus (Wang et al. 2011). The identified motifs in S. tuberosum DREB genes indicated high similarity with AP2 structure pattern. All conserved motifs had varied composition (Figs. S1-S2). Transcription factor domains and motifs are frequently linked to DNA binding, transcriptional activity, and protein interaction (Liu et al. 1999). The gene structure and motif analysis of the same subgroup were alike, thus validating the reliability of the phylogenetic tree classification. This finding was in line with the previous studies on DREB, which found that broad similarities existed in motifs and exon/intron structure between members of the same subgroup. 
411 Chromosomal positions demonstrated uneven scattering of 66 DREB genes over 12 potato

412 chromosomes. The asymmetrical arrangement of genes has been suggested to reveal information

413 about their evolution (Chen et al. 2016a). Whole genome duplications (WGD) or

414 polyploidization have been considered as the major causes of evolution that gives rise to novel

415 traits and new transcriptional regulatory sites that can alter expression patterns (Panchy et al.

416 2016). In total, 10 homologous pairs were confirmed to be produced by tandem duplication

417 events (Fig. 3). In contrast, eight paralogous gene pairs were produced through segmental

418 duplication events (Fig. 4). Moreover, gene pairs with tandem duplications were found on

419 chromosomes of the same origin. The most recent tandem duplication events were estimated to

420 be six million years ago for two pairs of genes belonging to A3 and A6 subgroup, respectively

421 (Data S6). S. spontaneum, $P$. trichocarpa, and soybean genome had undergone both tandem and

422 segmental duplications whereas only tandem duplications were found in P. vulgaris. Tandem

423 duplications are also known to be adaptively important in the development and function of

424 abiotic stress responsive genes. Previous studies reported that tandem repeats often share

425 common cis-acting elements, and may perform similar functions (Flagel \& Wendel 2009 ).

426 Hence, our study also emphasizes that StDREB tandem gene duplication pairs may share similar

427 functions and regulatory elements in their promoter region. To further investigate the potential

428 evolutionary mechanisms of the $S$. tuberosum DREB gene family, interspecies synteny was

429 inferred. The number of orthologous events of StDREB genes was greater with SIDREB genes

430 as compared to AtDREB genes, which may be due to close evolutionary link between $S$.

431 tuberosum and S. lycopersicum (Fig. 5). GO enrichment has been considered as a powerful tool

432 for enhancing the understanding of functional genomics and underlying molecular mechanism.

433 GO annotation verified the DNA-binding ability of StDREB genes which was consistent with the

434 findings in $P$. vulgaris (Fig. 6). All the StDREB genes were concentrated inside the nucleus.

435 Furthermore, our results from the subcellular localization also indicated that StDREB proteins

436 were primarily present inside the nucleus (Fig. 7).

437

438

439

440

441

442

443

444

445

446

447

448

449

450

451

452

453

454

\section{CONCLUSIONS}

In conclusion, we performed genome-wide identification and characterization of the DREB gene family in S. tuberosum and conducted a detailed investigation of their evolutionary relationship, genome organization, duplication events, and functional annotation using bioinformatics tools. In total, 66 DREB genes having an AP2 domain and conserved amino acid residues (Valine at position $14^{\text {th }}$ ) were identified in the Potato genome and unevenly mapped across 12 chromosomes. Based on the sequence alignment and phylogenetic analysis, StDREB genes were classified into six subgroups (A1-A6) corresponding to previous report of Arabidopsis. The results of gene structure and conserved motif analysis were found consistent with the phylogenetic classification. Gene structures and motif patterns showed that the StDREB members in the same subgroup displayed broad similarities. The expansion of the DREB gene family in Potato was aided greatly by tandem and segmental duplications. Evolutionary divergence analysis $(\mathrm{Ka} / \mathrm{Ks})$ suggested that the StDREBs were under strong purification selection during plant evolution. Synteny relationship analysis indicated that 35 and 46 StDREB genes were orthologous to Arabidopsis and S. lycopersicum, respectively. Furthermore, subcellular localization revealed that the StDREB genes were primarily located inside the nucleus. Through gene ontology (GO) annotation, we found most of the StDREB genes had DNA binding function, which suggested their role as important transcriptional activators. 
455

456

457

458

459

460

461

462

463

464

465

466

467

468

469

470

471

472

473

474

475

476

477

478

479

480

481

482

483

484

485

486

487

488

489

490

491

492

493

494

495

496

497

498

499

Functional enrichment indicated pivotal roles of StDREB genes in cell development, defense responses, and hormone signaling. Overall, our data delineated the evolutionary characteristics and genome duplication events along with biological and molecular functions of DREB genes in S. tuberosum. Taken together, our results will provide a foundation for unraveling the molecular mechanisms and further functional characterization of the StDREB gene family, thus providing sources for plant breeding and genetic engineering.

\section{REFERENCES}

Agarwal P, Agarwal PK, Nair S, Sopory S, and Reddy M. 2007. Stress-inducible DREB2A transcription factor from Pennisetum glaucum is a phosphoprotein and its phosphorylation negatively regulates its DNA-binding activity. Molecular Genetics and Genomics 277:189-198. DOI 10.1007/s00438-006-0183-z

Akhtar M, Jaiswal A, Taj G, Jaiswal J, Qureshi M, and Singh N. 2012. DREB1/CBF transcription factors: their structure, function and role in abiotic stress tolerance in plants. Journal of Genetics 91:385395. DOI 10.1007/s12041-012-0201-3

Allen MD, Yamasaki K, Ohme-Takagi M, Tateno M, and Suzuki MJTEj. 1998. A novel mode of DNA recognition by a $\beta$-sheet revealed by the solution structure of the GCC-box binding domain in complex with DNA. The EMBO Journal 17:5484-5496. DOI 10.1093/emboj/17.18.5484

Bailey TL, Johnson J, Grant CE, and Noble WS. 2015. The MEME suite. Nucleic acids research 43:W39W49. DOI 10.1093/nar/gkv416

Bouaziz D, Charfeddine M, Jbir R, Saidi MN, Pirrello J, Charfeddine S, Bouzayen M, and Gargouri-Bouzid R. 2015a. Identification and functional characterization of ten AP2/ERF genes in potato. Plant Cell, Tissue and Organ Culture (PCTOC) 123:155-172. DOI 10.1007/s11240-015-0823-2

Bouaziz D, Jbir R, Charfeddine S, Saidi MN, and Gargouri-Bouzid R. 2015b. The StDREB1 transcription factor is involved in oxidative stress response and enhances tolerance to salt stress. Plant Cell, Tissue and Organ Culture (PCTOC) 121:237-248. DOI 10.1007/s11240-014-0698-7

Bouaziz D, Pirrello J, Amor HB, Hammami A, Charfeddine M, Dhieb A, Bouzayen M, and Gargouri-Bouzid R. 2012. Ectopic expression of dehydration responsive element binding proteins (StDREB2) confers higher tolerance to salt stress in potato. Plant Physiology and Biochemistry 60:98-108. DOI 10.1016/j.plaphy.2012.07.029

Chai M, Cheng H, Yan M, Priyadarshani S, Zhang M, He Q, Huang Y, Chen F, Liu L, and Huang X. 2020. Identification and expression analysis of the DREB transcription factor family in pineapple (Ananas comosus (L.) Merr.). Peer J 8:e9006. DOI 10.7717\%2Fpeerj.9006

Charfeddine M, Charfeddine S, Bouaziz D, Messaoud RB, and Bouzid RG. 2017. The effect of cadmium on transgenic potato (Solanum tuberosum) plants overexpressing the StDREB transcription factors. Plant Cell, Tissue and Organ Culture (PCTOC) 128:521-541. DOI 10.1007/s11240-016-1130-2

Chen C, Chen H, Zhang Y, Thomas HR, Frank MH, He Y, and Xia R. 2020. TBtools: an integrative toolkit developed for interactive analyses of big biological data. Molecular plant 13:1194-1202. DOI 10.1016/j.molp.2020.06.009

Chen L, Han J, Deng X, Tan S, Li L, Li L, Zhou J, Peng H, Yang G, and He G. 2016a. Expansion and stress responses of AP2/EREBP superfamily in Brachypodium distachyon. Scientific Reports 6:1-14. https://doi.org/10.1038/srep21623

Chen M, Wang Q-Y, Cheng X-G, Xu Z-S, Li L-C, Ye X-G, Xia L-Q, and Ma Y-Z. 2007. GmDREB2, a soybean DRE-binding transcription factor, conferred drought and high-salt tolerance in transgenic plants. Biochemical and Biophysical Research Communications 353:299-305. DOI 10.1016/j.bbrc.2006.12.027

Peer] reviewing PDF | (2021:04:60076:1:0:NEW 20 May 2021) 
500

501

502

503

504

505

506

507

508

509

510

511

512

513

514

515

516

517

518

519

520

521

522

523

524

525

526

527

528

529

530

531

532

533

534

535

536

537

538

539

540

541

542

543

544

545

Chen Y, Huang L, Yan H, Zhang X, Xu B, and Ma X. 2016b. Cloning and characterization of an ABAindependent DREB transcription factor gene, HCDREB2, in Hemarthria compressa. Hereditas 153:1-7. DOI 10.1186/s41065-016-0008-y

Chen Y, Yang J, Wang Z, Zhang H, Mao X, and Li C. 2013. Gene structures, classification, and expression models of the DREB transcription factor subfamily in Populus trichocarpa. The Scientific World Journal 2013. DOI 10.1155/2013/954640

Conesa A, and Götz S. 2008. Blast2GO: a comprehensive suite for functional analysis in plant genomics. International journal of plant genomics 2008. DOI 10.1155/2008/619832

Dahal K, Li X-Q, Tai H, Creelman A, and Bizimungu B. 2019 Improving potato stress tolerance and tuber yield under a climate change scenario-a current overview. Frontiers in Plant Science 10:563. DOI $10.3389 /$ fpls.2019.00563

Davies Jr FT, Calderón CM, Huaman Z, and Gómez R. 2005. Influence of a flavonoid (formononetin) on mycorrhizal activity and potato crop productivity in the highlands of Peru. Scientia Horticulturae 106:318-329. DOI 10.1016/j.scienta.2005.04.013

Dietz K-J, Vogel MO, and Viehhauser A. 2010. AP2/EREBP transcription factors are part of gene regulatory networks and integrate metabolic, hormonal and environmental signals in stress acclimation and retrograde signalling. Protoplasma 245:3-14.

Dong C-J, and Liu J-Y. 2010. The Arabidopsis EAR-motif-containing protein RAP2. 1 functions as an active transcriptional repressor to keep stress responses under tight control. BMC Plant Biology 10:115. DOI 10.1186/1471-2229-10-47

Dossa K, Wei X, Li D, Fonceka D, Zhang Y, Wang L, Yu J, Boshou L, Diouf D, and Cissé N. 2016. Insight into the AP2/ERF transcription factor superfamily in sesame and expression profiling of DREB subfamily under drought stress. BMC Plant Biology 16:1-16.

Du H, Huang M, Zhang Z, and Cheng S. 2014. Genome-wide analysis of the AP2/ERF gene family in maize waterlogging stress response. Euphytica 198:115-126. DOI 10.1007/s10681-014-1088-2

Du X, Li W, Sheng L, Deng Y, Wang Y, Zhang W, Yu K, Jiang J, Fang W, and Guan Z. 2018. Over-expression of chrysanthemum CmDREB6 enhanced tolerance of chrysanthemum to heat stress. BMC Plant Biology 18:1-10. DOI 10.1186/s12870-018-1400-8

Dubouzet JG, Sakuma Y, Ito Y, Kasuga M, Dubouzet EG, Miura S, Seki M, Shinozaki K, and Yamaguchi-Shinozaki K. 2003. OsDREB genes in rice, Oryza sativa L., encode transcription activators that function in drought-, high-salt-and cold-responsive gene expression. The Plant Journal 33:751-763. DOI 10.1046/j.1365-313x.2003.01661.x

Egawa C, Kobayashi F, Ishibashi M, Nakamura T, Nakamura C, and Takumi S. 2006. Differential regulation of transcript accumulation and alternative splicing of a DREB2 homolog under abiotic stress conditions in common wheat. Genes \& Genetic Systems 81:77-91. DOI 10.1266/ggs.81.77

Feng Q, Jie L, Gui-You Z, Jun Z, Shou-Yi C, and Qiang L. 2003. Isolation and structural analysis of DREbinding transcription factor from maize (Zea mays L.). Journal of Integrative Plant Biology

45:331.

Flagel LE, and Wendel JF. 2009 Gene duplication and evolutionary novelty in plants. New Phytologist 183:557-564. DOI 10.1111/j.1469-8137.2009.02923.x

Gasteiger E, Gattiker A, Hoogland C, Ivanyi I, Appel RD, and Bairoch A. 2003. ExPASy: the proteomics server for in-depth protein knowledge and analysis. Nucleic acids research 31:3784-3788. DOI $10.1093 / \mathrm{nar} / \mathrm{gkg} 563$

Goodstein DM, Shu S, Howson R, Neupane R, Hayes RD, Fazo J, Mitros T, Dirks W, Hellsten U, and Putnam N. 2012. Phytozome: a comparative platform for green plant genomics. Nucleic acids research 40:D1178-D1186. DOI 10.1093/nar/gkr944

Peer] reviewing PDF | (2021:04:60076:1:0:NEW 20 May 2021) 
546

Guo A-Y, Zhu Q-H, Chen X, and Luo J-C. 2007. GSDS: a gene structure display server. Yi chuan= Hereditas 29:1023-1026. DOI 10.1360/yc-007-1023

Guo B, Wei Y, Xu R, Lin S, Luan H, Lv C, Zhang X, Song X, and Xu R. 2016. Genome-wide analysis of APETALA2/ethylene-responsive factor (AP2/ERF) gene family in barley (Hordeum vulgare L.). PLoS One 11:e0161322. DOI 10.1371/journal.pone.0161322

Guo J, and Wang M-H. 2011. Expression profiling of the DREB2 type gene from tomato (Solanum lycopersicum L.) under various abiotic stresses. Horticulture Environment and Biotechnology 52:105. DOI 10.1007/s13580-011-0125-5

Handayani T, Gilani SA, and Watanabe KN. 2019. Climatic changes and potatoes: How can we cope with the abiotic stresses? Breeding Science 69:545-563. DOI 10.1270/jsbbs.19070

He Y, Liu X, Ye L, Pan C, Chen L, Zou T, and Lu G. 2016. Genome-wide identification and expression analysis of two-component system genes in tomato. International Journal of Molecular Sciences 17:1204. DOI 10.3390/ijms17081204

Hichri I, Muhovski Y, Clippe A, Žižková E, Dobrev PI, Motyka V, and Lutts S. 2016. SIDREB2, a tomato dehydration-responsive element-binding 2 transcription factor, mediates salt stress tolerance in tomato and A rabidopsis. Plant, Cell \& Environment 39:62-79. DOI 10.1111/pce.12591

Hong J-P, and Kim WT. 2005. Isolation and functional characterization of the Ca-DREBLP1 gene encoding a dehydration-responsive element binding-factor-like protein 1 in hot pepper (Capsicum annuum L. cv. Pukang). Planta 220:875-888. DOI 10.1007/s00425-004-1412-5

Horton P, Park K-J, Obayashi T, Fujita N, Harada H, Adams-Collier C, and Nakai K. 2007. WoLF PSORT: protein localization predictor. Nucleic acids research 35:W585-W587. DOI 10.1093/nar/gkm259

Huala E, Dickerman AW, Garcia-Hernandez M, Weems D, Reiser L, LaFond F, Hanley D, Kiphart D, Zhuang M, and Huang W. 2001. The Arabidopsis Information Resource (TAIR): a comprehensive database and web-based information retrieval, analysis, and visualization system for a model plant. Nucleic acids research 29:102-105. DOI 10.1093/nar/29.1.102

Huang X, Song X, Chen R, Zhang B, Li C, Liang Y, Qiu L, Fan Y, Zhou Z, and Zhou H. 2020. Genome-wide analysis of the DREB subfamily in Saccharum spontaneum reveals their functional divergence during cold and drought stresses. Frontiers in Genetics 10:1326. DOI 10.3389/fgene.2019.01326

Hulo N, Bairoch A, Bulliard V, Cerutti L, De Castro E, Langendijk-Genevaux PS, Pagni M, and Sigrist CJ. 2006. The PROSITE database. Nucleic acids research 34:D227-D230. DOI 10.1093/nar/gkj063

Hussain T. 2016 Potatoes: ensuring food for the future. Advances in Plants \& Agriculture Research 3:178182. DOI 10.15406/apar.2016.03.00117

Hwang JE, Lim CJ, Chen H, Je J, Song C, and Lim CO. 2012. Overexpression of Arabidopsis dehydrationresponsive element-binding protein $2 \mathrm{C}$ confers tolerance to oxidative stress. Molecules and Cells 33:135-140. DOI 10.1007/s10059-012-2188-2

Jeffares DC, Penkett CJ, and Bähler J. 2008. Rapidly regulated genes are intron poor. Trends in Genetics 24:375-378. DOI 10.1016/j.tig.2008.05.006

Kimotho RN, Baillo EH, and Zhang Z. 2019. Transcription factors involved in abiotic stress responses in Maize (Zea mays L.) and their roles in enhanced productivity in the post genomics era. Peer J 7:e7211. DOI 10.7717/peerj.7211

Konzen ER, Recchia GH, Cassieri F, Caldas DGG, Berny Mier y Teran JC, Gepts P, and Tsai SM. 2019. DREB genes from common bean (Phaseolus vulgaris L.) show broad to specific abiotic stress responses and distinct levels of nucleotide diversity. International Journal of Genomics 2019. DOI $10.1155 / 2019 / 9520642$

Kudo M, Kidokoro S, Yoshida T, Mizoi J, Todaka D, Fernie AR, Shinozaki K, and Yamaguchi-Shinozaki K. 2017. Double overexpression of DREB and PIF transcription factors improves drought stress tolerance and cell elongation in transgenic plants. Plant Biotechnology Journal 15:458-471. DOI 10.1111\%2Fpbi.12644

Peer) reviewing PDF | (2021:04:60076:1:0:NEW 20 May 2021) 
594

595

596

597

598

599

600

601

602

603

604

605

606

607

608

609

610

611

612

613

614

615

616

617

618

619

620

621

622

623

624

625

626

627

628

629

630

631

632

633

634

635

636

637

638

639

640

Kumar S, Stecher G, Li M, Knyaz C, and Tamura K. 2018. MEGA X: molecular evolutionary genetics analysis across computing platforms. Molecular biology and evolution 35:1547-1549. DOI $10.1093 / \mathrm{molbev} / \mathrm{msy} 096$

Labbo AM, Mehmood M, Akhtar MN, Khan MJ, Tariq A, and Sadiq I. 2018. Genome-wide identification of AP2/ERF transcription factors in mungbean (Vigna radiata) and expression profiling of the VrDREB subfamily under drought stress. Crop and Pasture Science 69:1009-1019.

Lakhwani D, Pandey A, Dhar YV, Bag SK, Trivedi PK, and Asif MH. 2016. Genome-wide analysis of the AP2/ERF family in Musa species reveals divergence and neofunctionalisation during evolution. Scientific Reports 6:1-17. DOI 10.1038/srep18878

Lata C, and Prasad M. 2011. Role of DREBs in regulation of abiotic stress responses in plants. Journal of Experimental Botany 62:4731-4748. DOI 10.1093/jxb/err210

Letunic I, and Bork P. 2018. 20 years of the SMART protein domain annotation resource. Nucleic acids research 46:D493-D496. DOI 10.1093/nar/gkX922

Li P, Chai Z, Lin P, Huang C, Huang G, Xu L, Deng Z, Zhang M, Zhang Y, and Zhao X. 2020. Genome-wide identification and expression analysis of AP2/ERF transcription factors in sugarcane (Saccharum spontaneum L.). BMC Genomics 21:1-17. DOI 10.1186/s12864-020-07076-X

Liang Y, Kang K, Gan L, Ning S, Xiong J, Song S, Xi L, Lai S, Yin Y, and Gu J. 2019. Drought-responsive genes, late embryogenesis abundant group3 (LEA 3) and vicinal oxygen chelate, function in lipid accumulation in Brassica napus and Arabidopsis mainly via enhancing photosynthetic efficiency and reducing ROS. Plant Biotechnology Journal 17:2123-2142. DOI 10.1111/pbi.13127

Liu L, White MJ, and MacRae TH. 1999. Transcription factors and their genes in higher plants: functional domains, evolution and regulation. European Journal of Biochemistry 262:247-257. DOI 10.1046/j.1432-1327.1999.00349.x

Liu M, Sun W, Ma Z, Zheng T, Huang L, Wu Q, Zhao G, Tang Z, Bu T, and Li C. 2019. Genome-wide investigation of the AP2/ERF gene family in tartary buckwheat (Fagopyum Tataricum). BMC Plant Biology 19:1-19. DOI 10.1186/s12870-019-1681-6

Liu Q, Kasuga M, Sakuma Y, Abe H, Miura S, Yamaguchi-Shinozaki K, and Shinozaki K. 1998. Two transcription factors, DREB1 and DREB2, with an EREBP/AP2 DNA binding domain separate two cellular signal transduction pathways in drought-and low-temperature-responsive gene expression, respectively, in Arabidopsis. The Plant Cell 10:1391-1406. DOI $10.1105 /$ tpc.10.8.1391

Liu S, Wang X, Wang H, Xin H, Yang X, Yan J, Li J, Tran L-SP, Shinozaki K, and Yamaguchi-Shinozaki K. 2013. Genome-wide analysis of ZmDREB genes and their association with natural variation in drought tolerance at seedling stage of Zea mays L. PLoS Genetics 9:e1003790. DOI 10.1371/journal.pgen.1003790

Liu X, Zhu J, Wei C, Guo Q, Bian C, Xiang Z, and Zhao A. 2015. Genome-wide identification and characterization of the DREB transcription factor gene family in mulberry. Biologia Plantarum 59:253-265. DOI 10.1007/s10535-015-0498-x

Lucas S, Durmaz E, Akpınar BA, and Budak H. 2011 The drought response displayed by a DRE-binding protein from Triticum dicoccoides. Plant Physiology and Biochemistry 49:346-351. DOI 10.1016/j.plaphy.2011.01.016

Matsukura S, Mizoi J, Yoshida T, Todaka D, Ito Y, Maruyama K, Shinozaki K, and Yamaguchi-Shinozaki K. 2010. Comprehensive analysis of rice DREB2-type genes that encode transcription factors involved in the expression of abiotic stress-responsive genes. Molecular Genetics and Genomics 283:185-196. DOI 10.1007/s00438-009-0506-y

McKain MR, Tang H, McNeal JR, Ayyampalayam S, Davis JI, Depamphilis CW, Givnish TJ, Pires JC, Stevenson DW, and Leebens-Mack JH. 2016. A phylogenomic assessment of ancient polyploidy

Peer] reviewing PDF | (2021:04:60076:1:0:NEW 20 May 2021) 
641

642

643

644

645

646

647

648

649

650

651

652

653

654

655

656

657

658

659

660

661

662

663

664

665

666

667

668

669

670

671

672

673

674

675

676

677

678

679

680

681

682

683

684

685

686

687

and genome evolution across the Poales. Genome Biology and Evolution 8:1150-1164. DOI 10.1093\%2Fgbe\%2Fevw060

Mirzaei K, Bahramnejad B, and Fatemi S. 2020. Genome-wide identification and characterization of the bZIP gene family in potato (Solanum tuberosum). Plant Gene 24:100257. DOI 10.1016/j.plgene.2020.100257

Mistry J, Chuguransky S, Williams L, Qureshi M, Salazar GA, Sonnhammer EL, Tosatto SC, Paladin L, Raj S, and Richardson LJ. 2021. Pfam: The protein families database in 2021. Nucleic acids research 49:D412-D419. DOI 10.1093/nar/gkaa913

Movahedi S, Tabatabaei BS, Alizade H, Ghobadi C, Yamchi A, and Khaksar G. 2012. Constitutive expression of Arabidopsis DREB1B in transgenic potato enhances drought and freezing tolerance. Biologia Plantarum 56:37-42. DOI 10.1007/s10535-012-0013-6

Mueller LA, Solow TH, Taylor N, Skwarecki B, Buels R, Binns J, Lin C, Wright MH, Ahrens R, and Wang Y. 2005. The SOL Genomics Network. A comparative resource for Solanaceae biology and beyond. Plant physiology 138:1310-1317. DOI 10.1104/pp.105.060707

Niu X, Luo T, Zhao H, Su Y, Ji W, and Li H. 2020. Identification of wheat DREB genes and functional characterization of TaDREB3 in response to abiotic stresses. Gene 740:144514. DOI 10.1016/j.gene.2020.144514

Panchy N, Lehti-Shiu M, and Shiu S-H. 2016. Evolution of gene duplication in plants. Plant physiology 171:2294-2316. DOI 10.1104\%2Fpp.16.00523

Park S, Shi A, and Mou B. 2020. Genome-wide identification and expression analysis of the CBF/DREB1 gene family in lettuce. Scientific Reports 10:1-14. DOI 10.1038/s41598-020-62458-1

Rabara RC, Tripathi P, and Rushton PJ. 2014. The potential of transcription factor-based genetic engineering in improving crop tolerance to drought. OMICS: A Journal of Integrative Biology 18:601-614. DOI 10.1089\%2Fomi.2013.0177

Rae L, Lao NT, and Kavanagh TA. 2011. Regulation of multiple aquaporin genes in Arabidopsis by a pair of recently duplicated DREB transcription factors. Planta 234:429-444. DOI 10.1007/s00425-011$\underline{1414-z}$

Rao G, Sui J, Zeng Y, He C, and Zhang J. 2015. Genome-wide analysis of the AP2/ERF gene family in Salix arbutifolia. FEBS Open Bio 5:132-137. DOI 10.1016/i.fob.2015.02.002

Sakuma Y, Liu Q, Dubouzet JG, Abe H, Shinozaki K, and Yamaguchi-Shinozaki K. 2002. DNA-binding specificity of the ERF/AP2 domain of Arabidopsis DREBs, transcription factors involved in dehydration-and cold-inducible gene expression. Biochemical and Biophysical Research Communications 290:998-1009. DOI 10.1006/bbrc.2001.6299

Salih H, Odongo MR, Gong W, He S, and Du X. 2019. Genome-wide analysis of cotton C2H2-zinc finger transcription factor family and their expression analysis during fiber development. BMC Plant Biology 19:1-17. DOI 10.1186/s12870-019-2003-8

Sazegari S, and Niazi A. 2012. Isolation and molecular characterization of wheat ('Triticum aestivum') Dehydration Responsive Element Binding Factor (DREB) isoforms. Australian Journal of Crop Science 6:1037-1044.

Sharoni AM, Nuruzzaman M, Satoh K, Shimizu T, Kondoh H, Sasaya T, Choi I-R, Omura T, and Kikuchi S. 2011. Gene structures, classification and expression models of the AP2/EREBP transcription factor family in rice. Plant and Cell Physiology 52:344-360. DOI 10.1093/pcp/pcq196

Shen Y-G, Zhang W-K, He S-J, Zhang J-S, Liu Q, and Chen S-Y. 2003. An EREBP/AP2-type protein in Triticum aestivum was a DRE-binding transcription factor induced by cold, dehydration and ABA stress. Theoretical and Applied Genetics 106:923-930. DOI 10.1007/s00122-002-1131-x

Shi S, Zhang R, Zhao Z, Yang L, and Ge W. 2018. Genome-wide analysis of DREBs subfamily in foxtail millet. Genomics and Applied Biology 37:827-835. 
688

689

690

691

692

693

694

695

696

697

698

699

700

701

702

703

704

705

706

707

708

709

710

711

712

713

714

715

716

717

718

719

720

721

722

723

724

725

726

727

728

729

730

731

732

733

734

Shkolnik-Inbar D, and Bar-Zvi D. 2011. Expression of ABSCISIC ACID INSENSITIVE 4 (ABI4) in developing Arabidopsis seedlings. Plant Signaling \& Behavior 6:694-696. DOI 10.4161\%2Fpsb.6.5.14978

Shu Y, Liu Y, Zhang J, Song L, and Guo C. 2016. Genome-wide analysis of the AP2/ERF superfamily genes and their responses to abiotic stress in Medicago truncatula. Frontiers in Plant Science 6:1247. DOI 10.3389/fpls.2015.01247

Sun J, Peng X, Fan W, Tang M, Liu J, and Shen S. 2014. Functional analysis of BpDREB2 gene involved in salt and drought response from a woody plant Broussonetia papyrifera. Gene 535:140-149. DOI 10.1016/i.gene.2013.11.047

Vazquez-Hernandez M, Romero I, Escribano MI, Merodio C, and Sanchez-Ballesta MT. 2017. Deciphering the role of CBF/DREB transcription factors and dehydrins in maintaining the quality of table grapes cv. autumn royal treated with high $\mathrm{CO} 2$ levels and stored at 0 C. Frontiers in Plant Science 8:1591. DOI 10.3389\%2Ffpls.2017.01591

Voorrips R. 2002. MapChart: software for the graphical presentation of linkage maps and QTLs. Journal of heredity 93:77-78. DOI 10.1093/jhered/93.1.77

Wang L, Ma H, and Lin J. 2019. Angiosperm-wide and family-level analyses of AP2/ERF genes reveal differential retention and sequence divergence after whole-genome duplication. Frontiers in Plant Science 10:196. DOI 10.3389/fpls.2019.00196

Wang M, Zhuang J, Zou Z, Li Q, Xin H, and Li X. 2017. Overexpression of a Camellia sinensis DREB transcription factor gene (CSDREB) increases salt and drought tolerance in transgenic Arabidopsis thaliana. Journal of Plant Biology 60:452-461. DOI 10.1007/s12374-016-0547-9

Wang X, Chen X, Liu Y, Gao H, Wang Z, and Sun G. 2011. CkDREB gene in Caragana korshinskii is involved in the regulation of stress response to multiple abiotic stresses as an AP2/EREBP transcription factor. Molecular Biology Reports 38:2801-2811. DOI 10.1007/s11033-010-0425-3

Wendel JF, Jackson SA, Meyers BC, and Wing RA. 2016. Evolution of plant genome architecture. Genome Biology 17:1-14. DOI 10.1186/s13059-016-0908-1

Wu H, Lv H, Li L, Liu J, Mu S, Li X, and Gao J. 2015. Genome-wide analysis of the AP2/ERF transcription factors family and the expression patterns of DREB genes in Moso Bamboo (Phyllostachys edulis). PLoS One 10:e0126657. DOI 10.1371/journal.pone.0126657

Wu Z, Liang J, Zhang S, Zhang B, Zhao Q, Li G, Yang X, Wang C, He J, and Yi M. 2018. A canonical DREB2type transcription factor in lily is post-translationally regulated and mediates heat stress response. Frontiers in Plant Science 9:243. DOI 10.3389/fpls.2018.00243

Xianjun P, Xingyong M, Weihong F, Man S, Liqin C, Alam I, Lee B-H, Dongmei Q, Shihua S, and Gongshe L. 2011. Improved drought and salt tolerance of Arabidopsis thaliana by transgenic expression of a novel DREB gene from Leymus chinensis. Plant Cell Reports 30:1493-1502. DOI 10.1007/s00299$\underline{011-1058-2}$

Xie Z, Nolan T, Jiang H, Tang B, Zhang M, Li Z, and Yin Y. 2019. The AP2/ERF transcription factor TINY modulates brassinosteroid-regulated plant growth and drought responses in Arabidopsis. The Plant Cell 31:1788-1806. DOI 10.1105\%2Ftpc.18.00918

Yang Y, Wu J, Zhu K, Liu L, Chen F, and Yu D. 2009. Identification and characterization of two chrysanthemum (Dendronthemax moriforlium) DREB genes, belonging to the AP2/EREBP family. Molecular Biology Reports 36:71-81. DOI 10.1007/s11033-007-9153-8

Yu Z, Wang X, and Zhang L. 2018. Structural and functional dynamics of dehydrins: a plant protector protein under abiotic stress. International Journal of Molecular Sciences 19:3420. DOI 10.3390\%2Fijms19113420

Zhao K, Shen X, Yuan H, Liu Y, Liao X, Wang Q, Liu L, Li F, and Li T. 2013. Isolation and characterization of dehydration-responsive element-binding factor 2C (MsDREB2C) from Malus sieversii Roem. Plant and Cell Physiology 54:1415-1430. DOI 10.1093/pcp/pct087

Peer] reviewing PDF | (2021:04:60076:1:0:NEW 20 May 2021) 
Zhao T, Liang D, Wang P, Liu J, and Ma F. 2012. Genome-wide analysis and expression profiling of the DREB transcription factor gene family in Malus under abiotic stress. Molecular Genetics and Genomics 287:423-436. DOI 10.1007/s00438-012-0687-7

Zhao T, Xia H, Liu J, and Ma F. 2014. The gene family of dehydration responsive element-binding transcription factors in grape (Vitis vinifera): genome-wide identification and analysis, expression profiles, and involvement in abiotic stress resistance. Molecular Biology Reports 41:1577-1590. DOI 10.1007/s11033-013-3004-6

Zhou M-L, Ma J-T, Pang J-F, Zhang Z-L, Tang Y-X, and Wu Y-M. 2010. Regulation of plant stress response by dehydration responsive element binding (DREB) transcription factors. African Journal of Biotechnology 9:9255-9269. DOI 10.1093/jxb/erx118

Zhou W, Jia C-G, Wu X, Hu R-X, Yu G, Zhang X-H, Liu J-L, and Pan H-Y. 2016. ZmDBF3, a novel transcription factor from maize (Zea mays L.), is involved in multiple abiotic stress tolerance. Plant Molecular Biology Reporter 34:353-364. DOI 10.1007/s11105-015-0926-2

Zhou Y, Zhou W, Liu H, Liu P, and Li Z. 2020. Genome-wide analysis of the soybean DREB gene family: Identification, genomic organization and expression profiles in response to drought stress. Plant Breeding 139:1158-1167. DOI 10.1111/pbr.12867 
Figure 1

Phylogenetic tree of DREB proteins among Arabidopsis and S. tuberosum

StDREB proteins were assigned to six distinct subgroups (A1-A6) based on the classification of Arabidopsis. The six subgroups were highlighted with different colors.

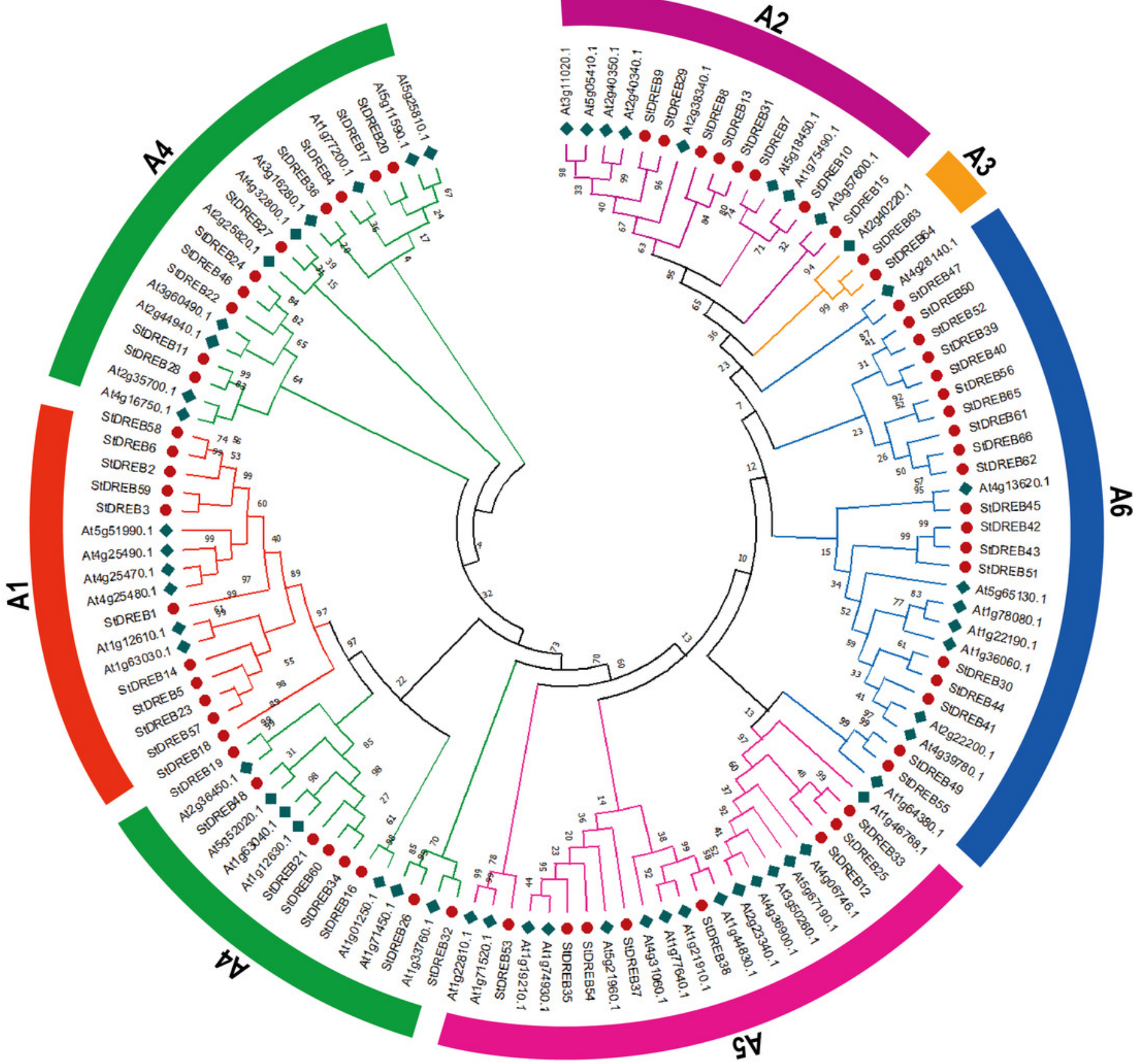




\section{Figure 2}

Analysis of phylogenetic evolutionary relationship, intron-exon organization and protein motif patterns of StDREBs

(A) Phylogenetic tree of 66 StDREB members was constructed with neighbor-joining method using Mega X. The 66 StDREB proteins were then classified into six subgroups (A1-A6). Gene structure was visualized using an online tool Gene Structure Display Server 2.0. The maroon boxes represented exons and the black line represented intron. The scale at the bottom showed the exon sizes. The numbers 0,1 , and 2 depicted the intron splicing phase. (C) Conserved motifs StDREB proteins were identified using MEME software. Fifteen predicted motifs were represented by distinct colored boxes and the grey lines indicated nonconserved regions. 


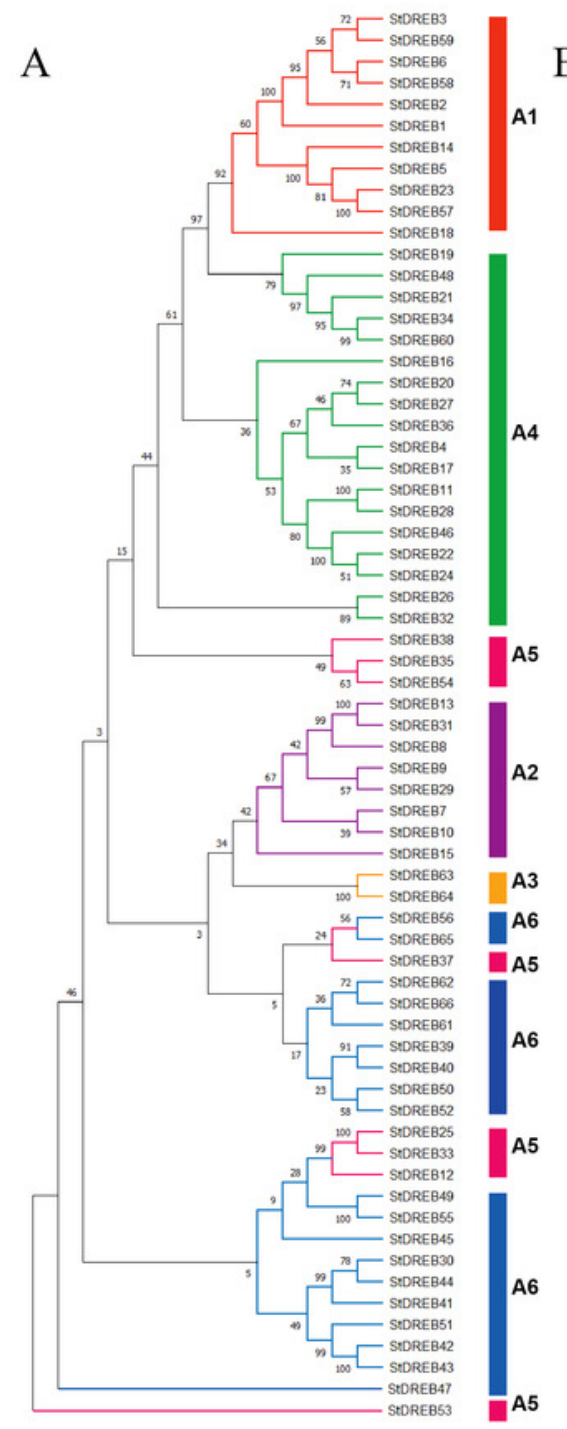

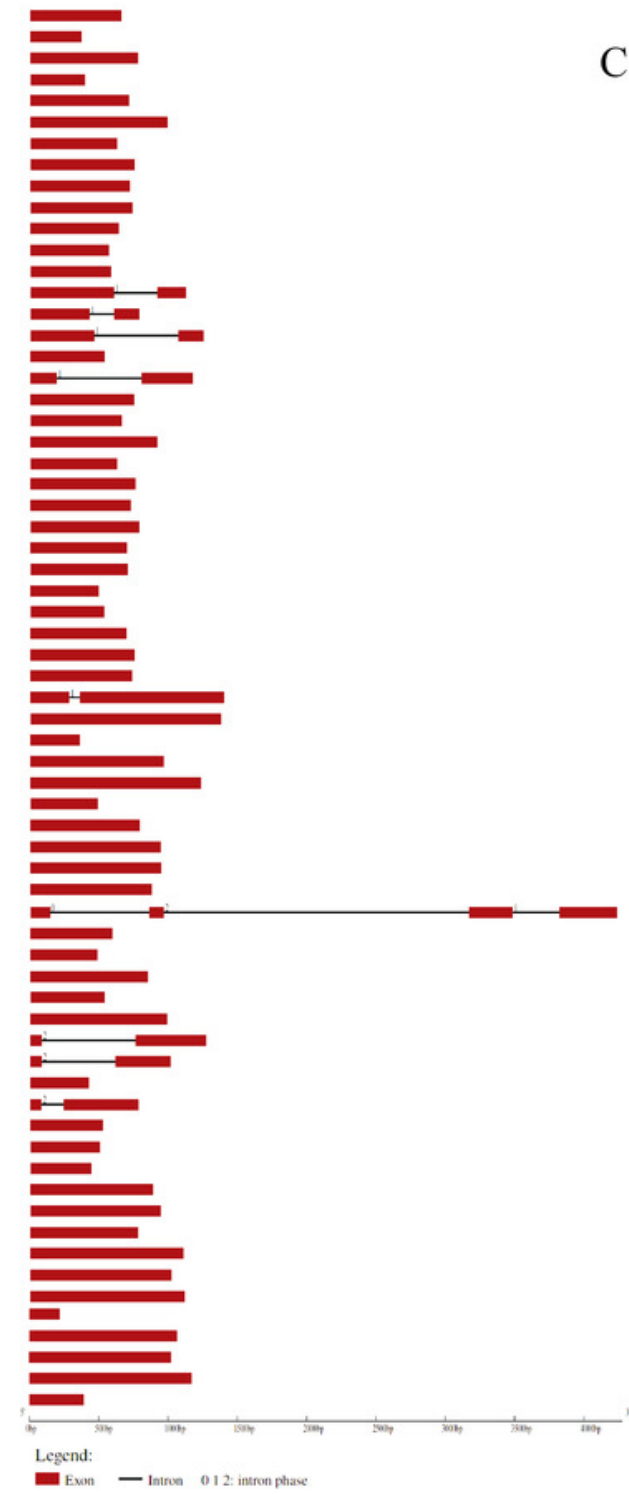

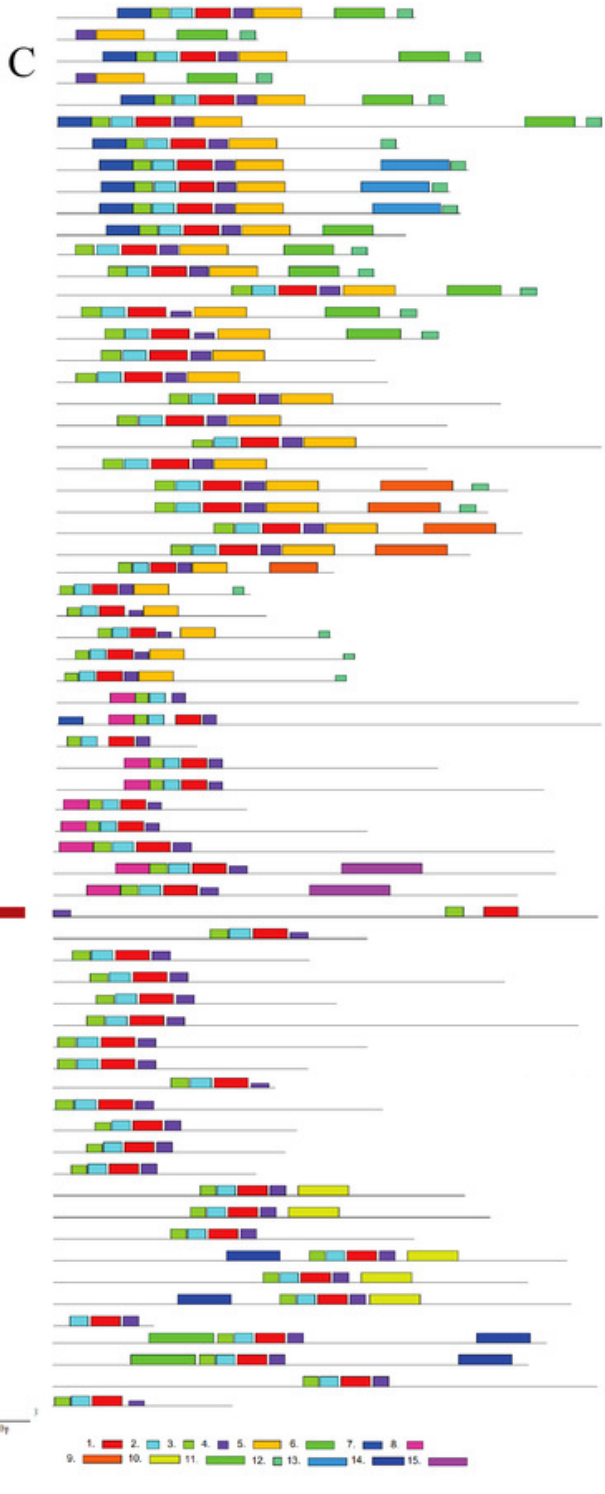


Figure 3

Genomic distribution of 66 StDREB genes across 12 potato chromosomes

Vertical bars indicated locus of StDREB genes on their respective chromosomes. The $\mathrm{x}$-axis scale represented the chromosome length while tandem duplicated gene pairs harbored by various chromosomes were indicated in red.
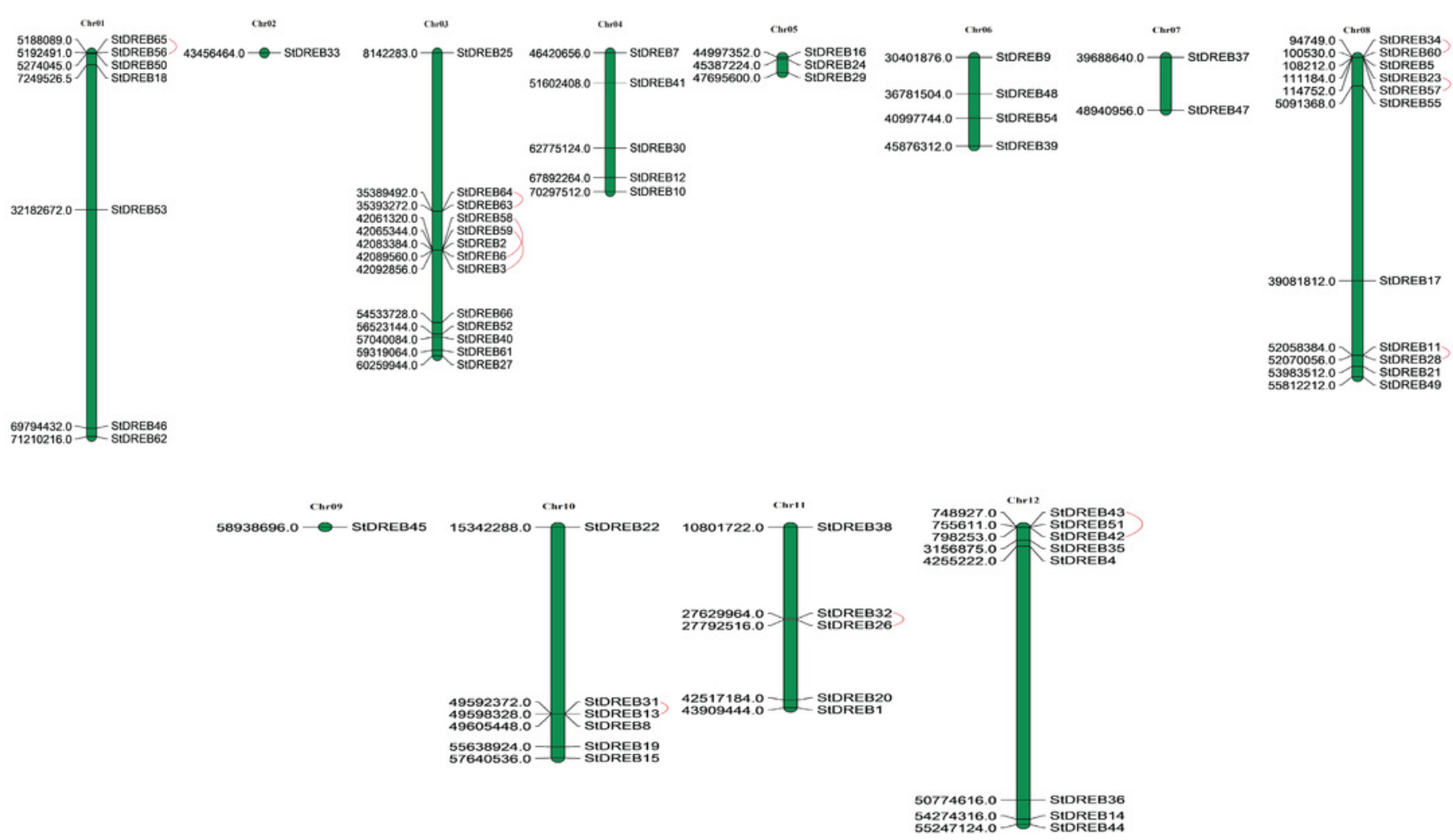
Figure 4

Segmental gene duplication events exhibited by StDREB genes across 12 potato chromosomes

Red lines represented segmental duplication of StDREB gene pairs. Chromosome number is located at top of each green block 


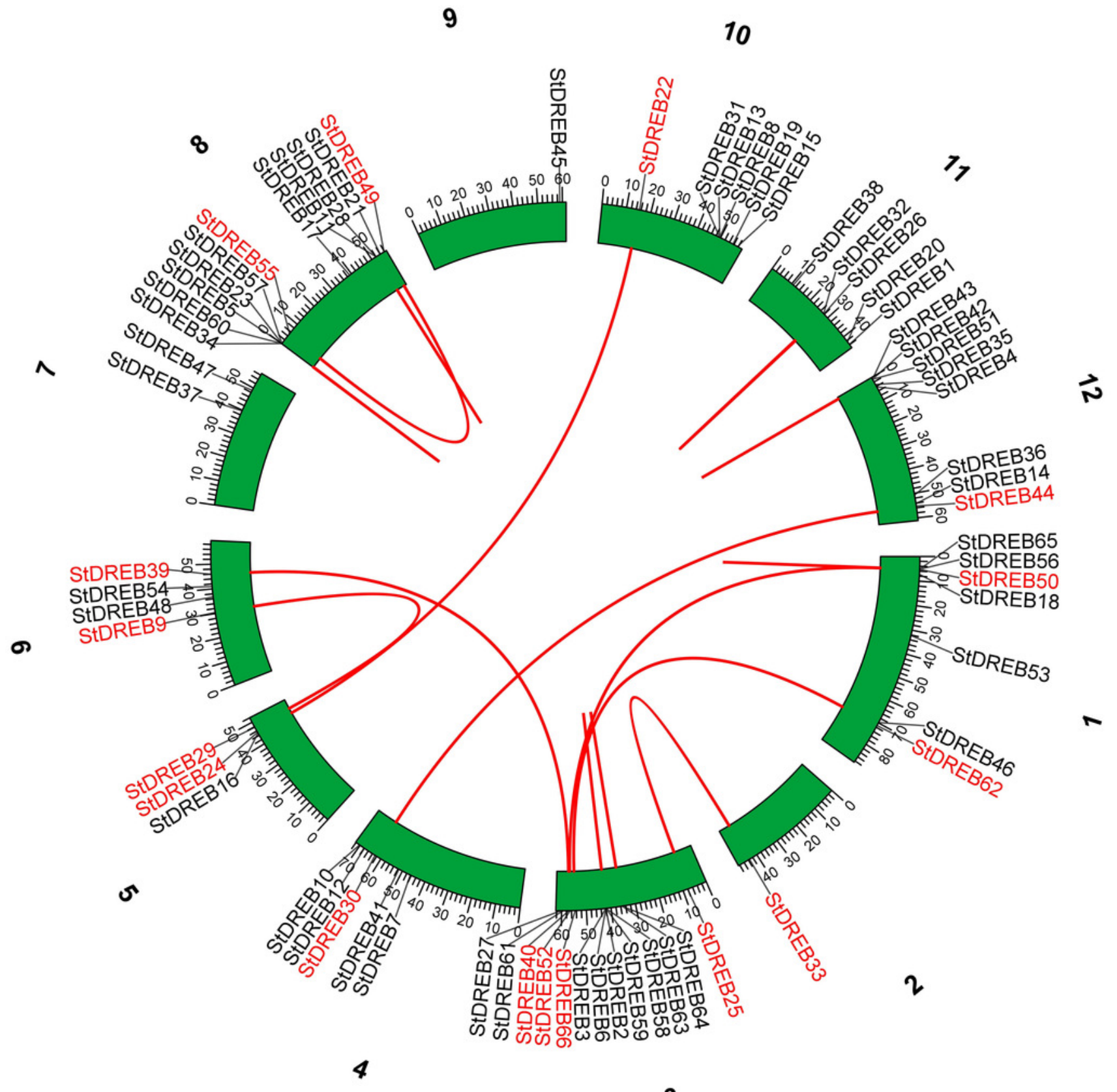


Figure 5

Synteny relationship analysis of DREB genes between $S$. tuberosum, $A$. thaliana and $S$. lycopersicum

Red and green bars represented the chromosomes while red lines indicated the homology and evolutionary link of StDREB genes with $A$. thaliana and S. lycopersicum, respectively. In addition, grey lines indicated all the collinear blocks present in their respective genomes. 

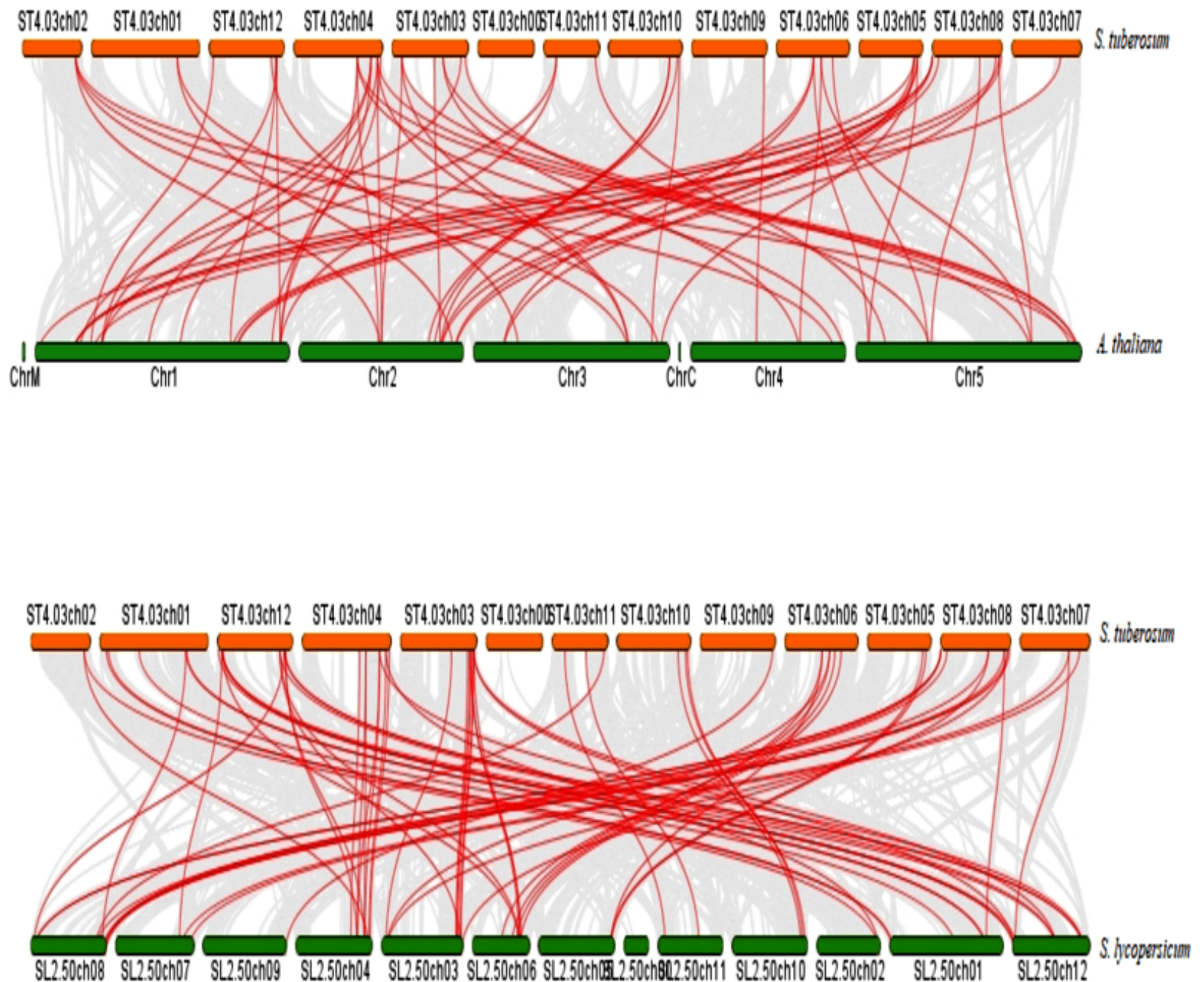
Figure 6

Gene Ontology (GO) and functional enrichment analysis of StDREB genes by using Blast2GO software

(A) GO distribution by level processes. (B) Direct count GO. Green bars represented biological processes (BP), blue bars represented molecular functions (MF), and yellow bars represented cellular compartments (CC) 
(a)

Biological processes

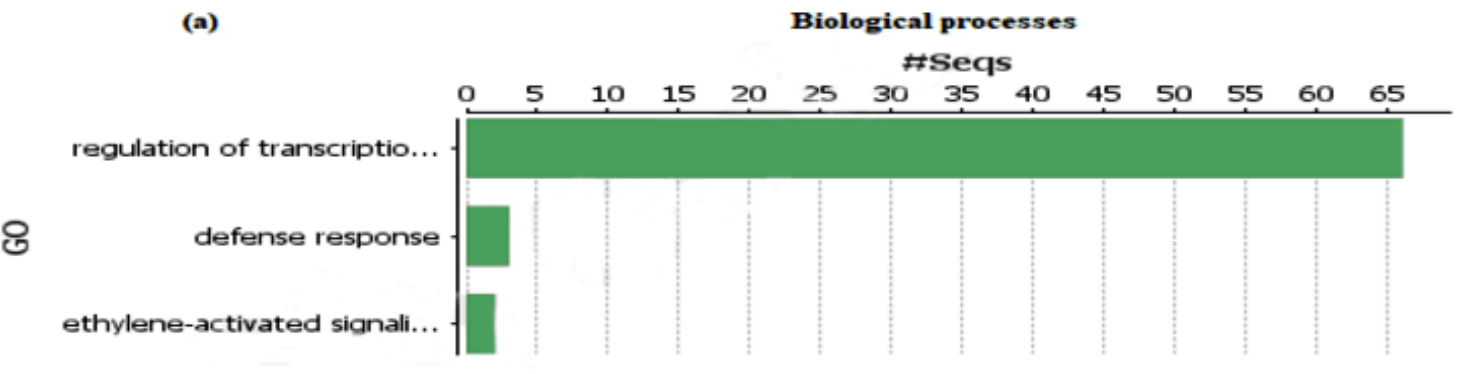

Molecular Functions
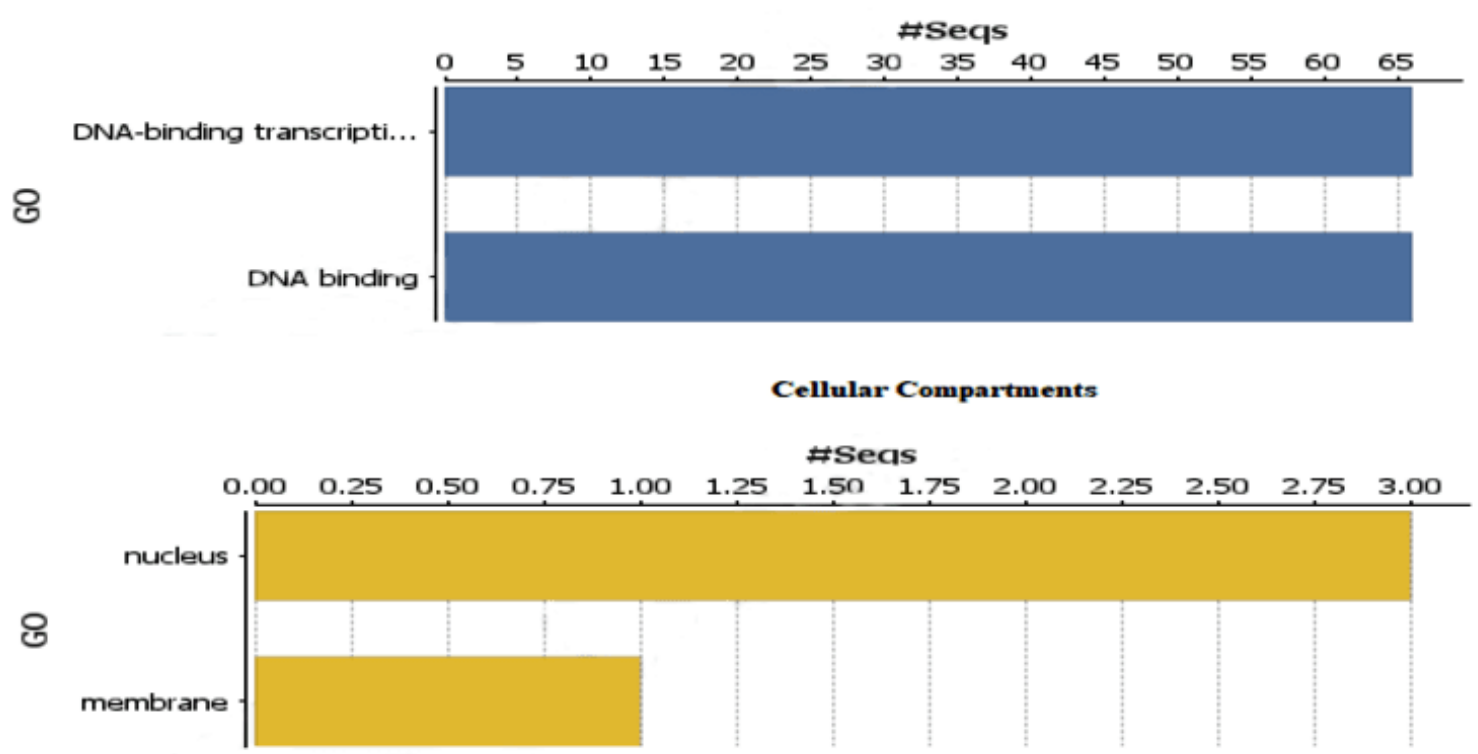

(b)

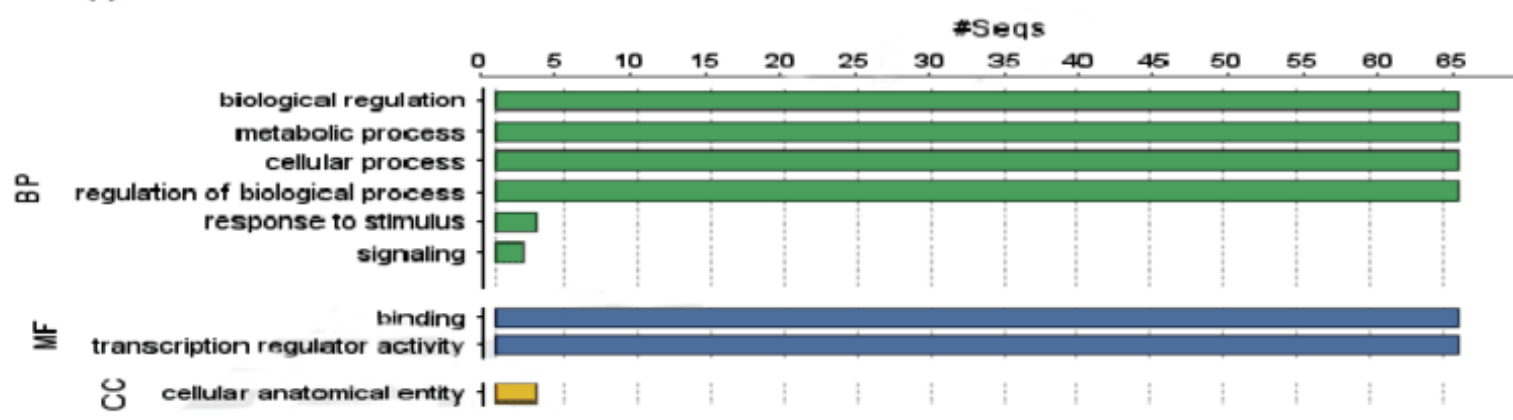


Figure 7

Heat map of subcellular localization predictions for 66 StDREB proteins

The color scale represents abundance of StDREBs in various cellular compartments. 


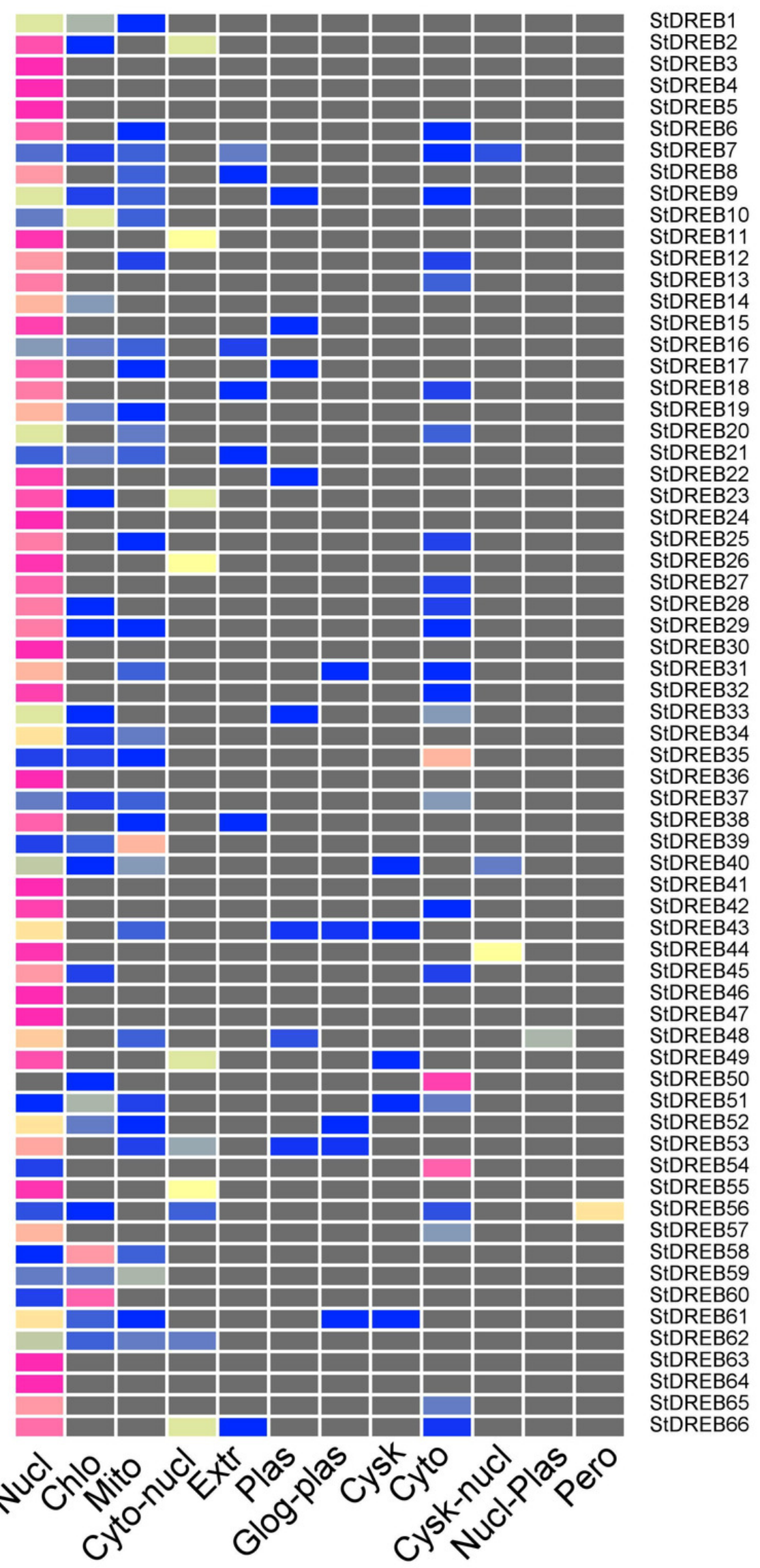

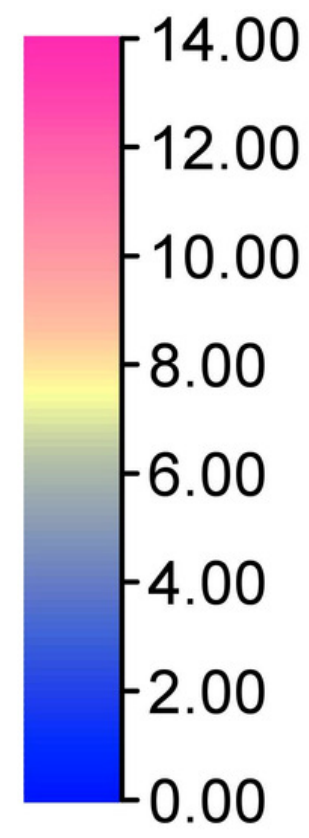




\section{Table 1 (on next page)}

Conserved AP2 domain sequences from six representative StDREB genes 


\section{Table 1:}

2 AP2 domain sequences identified in StDREB genes by Pfam. Different colors indicated alignment of 3 the AP2 domain. First row depicted consensus region of the Hidden Markov Model (HMM); second row 4 depicted the match between query protein sequences and HMM; third row demonstrated the degree of 5 confidence in each individually aligned residue; whereas fourth row demonstrated the query sequence.

\begin{tabular}{|c|c|c|c|}
\hline Gene ID & PF00847 AP2 Domain & $\begin{array}{c}\text { Sequence } \\
\text { Alignment }\end{array}$ & $\begin{array}{r}\text { HMM } \\
\text { Length }\end{array}$ \\
\hline StDREB3 & 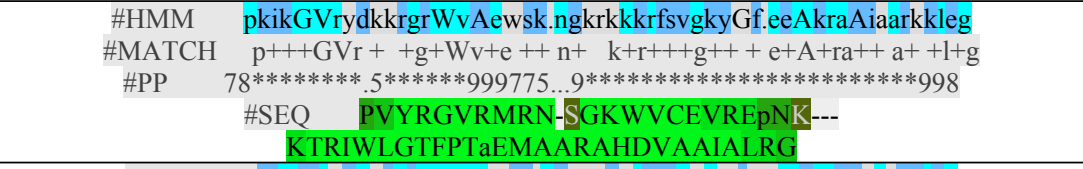 & $60-110$ & 54 \\
\hline StDREB12 & 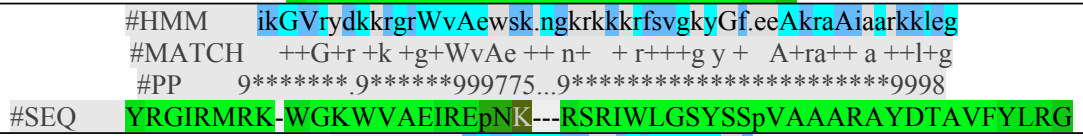 & $18-66$ & 54 \\
\hline StDREB13 & $\begin{array}{cc}\text { \#HMM } & \text { pkikGVrydkkrgrWvAews } \\
\text { \#MATCH } & +\mathrm{k}++\mathrm{GVr}+++\mathrm{g}+\text { WvAe }+ \\
\text { \#PP } & 79 * * * * * * * .9 * * * * * 65 \\
\text { \#SEQ } & \text { CKYRGVRQRT-WGKWVAEIR } \\
\end{array}$ & $69-87$ & 54 \\
\hline StDREB28 & 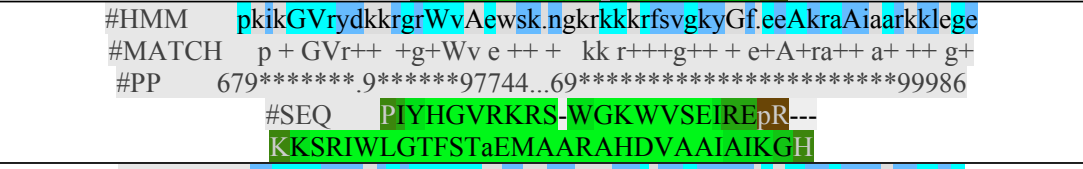 & $58-109$ & 54 \\
\hline StDREB47 & $\begin{array}{cc}\text { \#HMM } & \text { ikGVrydkkrgrWvAe...wskngkrkkkrfsvgkyGf.eeAkraAiaarkkleg } \\
\text { \#MATCH } & ++\mathrm{GVr}++++\mathrm{g}+\text { WvAe }+++++\mathrm{r}++\mathrm{g}++++\mathrm{e} A++\mathrm{a}+++++\mathrm{kl}+\mathrm{g} \\
\text { \#PP } & 9 * * * * * * * .9 * * * * * * 5554 . .55 \ldots . .8 * * * * * * * * * * * * * * * * * * * 9999987 \\
& \text { \#SEQ YRGVRQRH-WGKWVAEirIP--RN--- } \\
& \text { RTRLWLGTFDTaEDAAMAYDREAYKLRG }\end{array}$ & $184-232$ & 54 \\
\hline StDREB63 & $\begin{array}{lll} & \text { \#HMM } & \text { kikGVrydkkrgrWvAewsk.ngkrkkkrfsvgkyGf.eeAkraAiaark } \\
& \text { \#MATCH } & +++\mathrm{GVr}+++\mathrm{g}+\mathrm{WvAe}+++\mathrm{k}++\mathrm{r}++\mathrm{g}+++\mathrm{e} \mathrm{A}+\mathrm{ra}+++\mathrm{a}+ \\
& \text { \#PP } & 69 * * * * * * * .9 * * * * * * 87744 \ldots 69 * * * * * * * * * * * * * * * * * * * * 996 \\
\text { \#SEQ } & \text { RYRGVRQRS-WGKWVAEIREpR---KRTRRWLGTFATaEDAARAYDRAAI } \\
\end{array}$ & $64-109$ & 54 \\
\hline
\end{tabular}

6

7

8 